\title{
Pseudoduality Between Symmetric Space Sigma Models
}

\author{
Mustafa Sarisaman* \\ Department of Physics \\ University of Miami \\ P.O. Box 248046 \\ Coral Gables, FL 33124 USA
}

Monday, April 27, 2009

\begin{abstract}
We study the pseudoduality transformation on the symmetric space sigma models. We switch the Lie group valued pseudoduality equations to Lie algebra valued ones, which leads to an infinite number of pseudoduality equations. We obtain an infinite number of conserved currents on the tangent bundle of the pseudodual manifold. We show that there can be mixing of decomposed spaces with each other, which leads to mixings of the following expressions. We obtain the mixing forms of curvature relations and one loop renormalization group beta functions by means of these currents.
\end{abstract}

*msarisaman@physics.miami.edu 


\section{Introduction}

We know that there is a well defined duality transformation 11 between target spaces of the sigma models on symmetric spaces with opposite curvatures which preserves the stress energy tensors associated with each sigma models though it is not a canonical transformation. In this paper we present the general solution of the pseudoduality equations [5] between two symmetric space sigma models, and construct the pseudodual currents by means of these equations. We will do our calculations regarding $G$ as a symmetric space $G \times G / G$, and then extend our construction using Cartan's decomposition of symmetric spaces. We will use the references [6, 7, 8, 9] for the symmetric space construction, and utilize the literature [10, 11, 12, 13, 14, 15, 16, 17, 18, 19, 20, 21, 22, 23. on various applications to sigma models. Since pseudoduality is defined on spacetime coordinates [4], and is best done on the orthonormal coframes bundle $2 S O(M)$, we leave this construction to later [24]. In this paper we will do our calculations on the pullback bundle of target space $M$. Hence pulling structures back to spacetime is implicit, and not emphasized. We will see that this construction will give us complicated expressions for $T$ as opposed to the simplified form (identity) on $S O(M)$ [24].

\section{Pseudoduality Between strict WZW Mod- els}

We consider a strict WZW sigma model 25] based on a compact Lie group of dimension $n$. Lagrangian [25, 14, 21, 22, 23] for this model is defined by

$$
\mathcal{L}=\frac{1}{2} \operatorname{Tr}\left(g^{-1} \partial_{\mu} g g^{-1} \partial^{\mu} g\right)+\Gamma
$$

where $\Gamma$ represents the WZ term, and the field $g$ is given by the map $g$ : $\Sigma \rightarrow G$. We take $\Sigma$ to be two dimensional Minkowski space, and $\sigma^{ \pm}=$ $\tau \pm \sigma$ is the standard lightcone coordinates as above. There is a global continuous symmetry $G_{L} \times G_{R}$ which gives us the conserved currents $J_{+}^{(L)}=$ $g_{L}^{-1} \partial_{+} g_{L}$ and $J_{-}^{(R)}=\left(\partial_{-} g_{R}\right) g_{R}^{-1}$ taking values in the Lie algebra of $G$, and $g=g_{R}\left(\sigma^{-}\right) g_{L}\left(\sigma^{+}\right)$is the solution giving the invariance of these currents.

\footnotetext{
${ }^{1}$ This transformation is known as pseudoduality transformation [1, 2, 3, 4]

${ }^{2} S O(M)=M \times S O(n)$, where $\operatorname{dim}(M)=n$.
} 
The equations of motion following from (11) correspond to the conservation of these currents:

$$
\partial_{-}\left(g_{L}^{-1} \partial_{+} g_{L}\right)=\partial_{+}\left[\left(\partial_{-} g_{R}\right) g_{R}^{-1}\right]=0
$$

Let $\tilde{G}$ be compact Lie group of the same dimension as $G$, and $\tilde{g}: \Sigma \rightarrow \tilde{G}$. Equations of motion are given by

$$
\partial_{-}\left({\tilde{g_{L}}}^{-1} \partial_{+} \tilde{g_{L}}\right)=\partial_{+}\left[\left(\partial_{-} \tilde{g_{R}}\right){\tilde{g_{R}}}^{-1}\right]=0
$$

Solutions of equations of motion for both models can be combined in pseudoduality equations as

$$
\begin{aligned}
& \left(\tilde{g}^{-1} \partial_{+} \tilde{g}\right)^{i}=T_{j}^{i}\left(g^{-1} \partial_{+} g\right)^{j} \\
& \left(\tilde{g}^{-1} \partial_{-} \tilde{g}\right)^{i}=-T_{j}^{i}\left(g^{-1} \partial_{-} g\right)^{j}
\end{aligned}
$$

where $T$ is an orthogonal matrix connecting target space elements $g^{-1} d g$ and $\tilde{g}^{-1} d \tilde{g}$.

Taking $\partial_{-}$of first equation (4) with the help of equations of motions (2) and (3) shows that $T$ is a function of $\sigma^{+}$only. Taking $\partial_{+}$of second equation (5) gives us the following differential equation

$$
\left[\left(\partial_{+} T\right) T^{-1}\right]_{j}^{i}=f_{m l}^{k} T_{k}^{i} T_{l}^{j}\left(g_{L}^{-1} \partial_{+} g_{L}\right)^{m}-\tilde{f}_{k j}^{i} T_{l}^{k}\left(g_{L}^{-1} \partial_{+} g_{L}\right)^{l}
$$

We suggest an exponential solution $3 T=e^{X}$, and use the result [6, 10, 12]

$$
\left(\partial_{+} T\right) T^{-1}=-\frac{1-e^{a d X}}{a d X} \partial_{+} X=\sum_{n=0}^{\infty} \frac{1}{(n+1) !}\left[X, \ldots,\left[X, \partial_{+} X\right]\right]
$$

where $a d X: \mathbf{g} \rightarrow \mathbf{g}$, the adjoint representation of $\mathrm{X}$, and $\operatorname{adX}(Y)=[X, Y]$ $\forall Y \epsilon \mathrm{g}$. We let $X \rightarrow \varepsilon X$ and look for a perturbation solution, and hence the left-hand side of equation (6) is

$$
\left[\left(\partial_{+} T\right) T^{-1}\right]_{j}^{i}=\varepsilon\left(\partial_{+} X\right)_{j}^{i}+\frac{\varepsilon^{2}}{2}\left[X, \partial_{+} X\right]_{j}^{i}+\frac{\varepsilon^{3}}{3 !}\left[X,\left[X, \partial_{+} X\right]\right]+\ldots
$$

We insert an order parameter $\varepsilon$ to the right-hand side of (6), and get

$$
\begin{aligned}
{\left[\left(\partial_{+} T\right) T^{-1}\right]_{j}^{i}=} & \varepsilon f_{m l}^{k} T_{k}^{i} T_{l}^{j}\left(g_{L}^{-1} \partial_{+} g_{L}\right)^{m}-\varepsilon \tilde{f}_{k j}^{i} T_{l}^{k}\left(g_{L}^{-1} \partial_{+} g_{L}\right)^{l} \\
= & \varepsilon f_{m l}^{k}(1+\varepsilon X)_{k}^{i}(1+\varepsilon X)_{l}^{j}\left(g_{L}^{-1} \partial_{+} g_{L}\right)^{m}-\varepsilon \tilde{f}_{k j}^{i}(1+\varepsilon X)_{l}^{k}\left(g_{L}^{-1} \partial_{+} g_{L}\right)^{m} \\
= & \varepsilon f_{m j}^{i}\left(g_{L}^{-1} \partial_{+} g_{L}\right)^{m}-\varepsilon \tilde{f}_{k j}^{i}\left(g_{L}^{-1} \partial_{+} g_{L}\right)^{k}+\varepsilon^{2} f_{m l}^{i} X_{l}^{j}\left(g_{L}^{-1} \partial_{+} g_{L}\right)^{m} \\
& +\varepsilon^{2} f_{m j}^{k} X_{k}^{i}\left(g_{L}^{-1} \partial_{+} g_{L}\right)^{m}-\varepsilon^{2} \tilde{f}_{k j}^{i} X_{l}^{k}\left(g_{L}^{-1} \partial_{+} g_{L}\right)^{l}+\mathcal{O}\left(\varepsilon^{3}\right)
\end{aligned}
$$

\footnotetext{
${ }^{3}$ We notice that $X \in \operatorname{so}(n)$, the Lie algebra of $S O(n)$
} 
Comparing (8) and (9) in the first order of $\varepsilon$ gives us

$$
\left(\partial_{+} X\right)_{j}^{i}=\left(f_{k j}^{i}-\tilde{f}_{k j}^{i}\right)\left(g_{L}^{-1} \partial_{+} g_{L}\right)^{k}
$$

This leads to the solution

$$
X_{j}^{i}=X(0)_{j}^{i}+\left(f_{k j}^{i}-\tilde{f}_{k j}^{i}\right) \int_{0}^{\sigma^{+}}\left(g_{L}^{-1} \partial_{+} g_{L}\right)^{k} d \sigma^{\prime+}
$$

Hence the matrix $T$ may be written as

$$
T_{j}^{i}=\delta_{j}^{i}+X(0)_{j}^{i}+\left(f_{k j}^{i}-\tilde{f}_{k j}^{i}\right) \int_{0}^{\sigma^{+}}\left(g_{L}^{-1} \partial_{+} g_{L}\right)^{k} d \sigma^{\prime+}
$$

We see that if both sigma models based on the same groups, i.e $G=\tilde{G}$, target space of transformed model will be globally shifted as determined by the tangent space of unit element of $T$. We set $X(0)_{j}^{i}$ equal to zero.

Now we plug this in the pseudoduality equations (4) and (5) to find fields $\tilde{g}^{-1} \partial_{+} \tilde{g}$ and $\tilde{g}^{-1} \partial_{-} \tilde{g}$ which lead us to construct the pseudodual currents. We switch from Lie group-valued fields to the lie algebra-valued fields, and we let 4 g $g=e^{Y}$ and $\tilde{g}=e^{\tilde{Y}}$. Using the result [6, 10, 12]

$$
e^{-X} \partial_{\mu} e^{X}=\frac{1-e^{-a d X}}{a d X} \partial_{\mu} X=\sum_{k=0}^{\infty} \frac{(-1)^{k}}{(k+1) !}\left[X, \ldots,\left[X, \partial_{\mu} X\right]\right]
$$

we can write the following

$$
\begin{gathered}
g_{L}^{-1} \partial_{+} g_{L}=\partial_{+} Y_{L}-\frac{1}{2 !}\left[Y_{L}, \partial_{+} Y_{L}\right]+\frac{1}{3 !}\left[Y_{L},\left[Y_{L}, \partial_{+} Y_{L}\right]\right]+\ldots \\
g^{-1} \partial_{-} g=\partial_{-} Y_{R}-\left[Y_{L}, \partial_{-} Y_{R}\right]-\frac{1}{2}\left[Y_{R}, \partial_{-} Y_{R}\right]+\frac{1}{2}\left[Y_{L},\left[Y_{R}, \partial_{-} Y_{R}\right]\right] \\
+\frac{1}{2}\left[Y_{L},\left[Y_{L}, \partial_{-} Y_{R}\right]\right]+\frac{1}{6}\left[Y_{R},\left[Y_{R}, \partial_{-} Y_{R}\right]\right] \ldots
\end{gathered}
$$

and the equations of motion for the left and right currents will be

$$
\partial_{-}\left(g_{L}^{-1} \partial_{+} g_{L}\right)=\partial_{+-}^{2} Y_{L}-\frac{1}{2 !} \partial_{-}\left[Y_{L}, \partial_{+} Y_{L}\right]+\frac{1}{3 !} \partial_{-}\left[Y_{L},\left[Y_{L}, \partial_{+} Y_{L}\right]\right]+\ldots=0
$$

\footnotetext{
${ }^{4} Y$ is the lie algebra of $g, Y \in \mathbf{g}$.
} 


$$
\partial_{+}\left[\left(\partial_{-} g_{R}\right) g_{R}^{-1}\right]=\partial_{+-}^{2} Y_{R}+\frac{1}{2 !} \partial_{+}\left[Y_{R}, \partial_{-} Y_{R}\right]+\frac{1}{3 !} \partial_{+}\left[Y_{R},\left[Y_{R}, \partial_{-} Y_{R}\right]\right]+\ldots=0
$$

where $g_{L / R}=e^{Y_{L / R}}$, and we used equation (17). We may write similar equations with tilde $(\sim)$. Hence transformation matrix $T$ (12) will be

$$
T_{j}^{i}=\delta_{j}^{i}+\left(f_{k j}^{i}-\tilde{f}_{k j}^{i}\right) Y_{L}^{k}-\frac{1}{2 !}\left(f_{k j}^{i}-\tilde{f}_{k j}^{i}\right) \int_{0}^{\sigma^{+}}\left[Y_{L}, \partial_{+} Y_{L}\right]^{k} d \sigma^{\prime+}
$$

We impose a solution $Y=\sum_{n=1}^{\infty} \varepsilon^{n} y_{n}$ to determine the nonlinear parts of the equations (14) and (15) in terms of $\varepsilon$, where $\varepsilon$ is a small parameter. Thus transformation matrix (18) becomes

$T_{j}^{i}=\delta_{j}^{i}+\varepsilon\left(f_{k j}^{i}-\tilde{f}_{k j}^{i}\right) y_{L 1}^{k}+\varepsilon^{2}\left(f_{k j}^{i}-\tilde{f}_{k j}^{i}\right)\left[y_{L 2}^{k}-\frac{1}{2} \int_{0}^{\sigma^{+}}\left[y_{L 1}, \partial_{+} y_{L 1}\right]^{k} d \sigma^{\prime+}\right]+\mathcal{O}\left(\varepsilon^{3}\right)$

and we have the following expressions for (14) and (15)

$$
\begin{aligned}
g_{L}^{-1} \partial_{+} g_{L} & =\varepsilon \partial_{+} y_{L 1}+\varepsilon^{2}\left(\partial_{+} y_{L 2}-\frac{1}{2}\left[y_{L 1}, \partial_{+} y_{L 1}\right]\right) \\
& +\varepsilon^{3}\left(\partial_{+} y_{L 3}-\frac{1}{2}\left[y_{L 1}, \partial_{+} y_{L 2}\right]-\frac{1}{2}\left[y_{L 2}, \partial_{+} y_{L 1}\right]+\frac{1}{6}\left[y_{L 1},\left[y_{L 1}, \partial_{+} y_{L 1}\right]\right]\right)+H . O(\varepsilon) \\
g^{-1} \partial_{-} g & =\varepsilon \partial_{-} y_{R 1}+\varepsilon^{2}\left(\partial_{-} y_{R 2}-\left[y_{L 1}, \partial_{-} y_{R 1}\right]-\frac{1}{2}\left[y_{R 1}, \partial_{-} y_{R 1}\right]\right) \\
& +\varepsilon^{3}\left(\partial_{-} y_{R 3}-\left[y_{L 2}, \partial_{-} y_{R 1}\right]-\left[y_{L 1}, \partial_{-} y_{R 2}\right]-\frac{1}{2}\left[y_{R 2}, \partial_{-} y_{R 1}\right]-\frac{1}{2}\left[y_{R 1}, \partial_{-} y_{R 2}\right]\right. \\
& \left.+\frac{1}{2}\left[y_{L 1},\left[y_{R 1}, \partial_{-} y_{R 1}\right]\right]+\frac{1}{2}\left[y_{L 1},\left[y_{L 1}, \partial_{-} y_{R 1}\right]\right]\right)+H . O(\varepsilon)
\end{aligned}
$$

Therefore first pseudoduality equation (4) can be split into infinite number of equations, determined by each order of $\varepsilon$ as follows, 
(1.i) $\partial_{+} \tilde{y}_{L 1}^{i}=\partial_{+} y_{L 1}^{i}$

(1.ii) $\partial_{+} \tilde{y}_{L 2}^{i}+\frac{1}{2}\left[\tilde{y}_{L 1}, \partial_{+} \tilde{y}_{L 1}\right]_{\tilde{G}}^{i}=\partial_{+} y_{L 2}^{i}+\frac{1}{2}\left[y_{L 1}, \partial_{+} y_{L 1}\right]_{G}^{i}$

(1.iii) $\partial_{+} \tilde{y}_{3}^{i}-\frac{1}{2}\left[\tilde{y}_{1}, \partial_{+} \tilde{y}_{2}\right]_{\tilde{G}}^{i}-\frac{1}{2}\left[\tilde{y}_{2}, \partial_{+} \tilde{y}_{1}\right]_{\tilde{G}}^{i}+\frac{1}{6}\left[\tilde{y}_{1},\left[\tilde{y}_{1}, \partial_{+} \tilde{y}_{1}\right]_{\tilde{G}}\right]_{\tilde{G}}^{i}=\partial_{+} y_{3}^{i}$

$+\frac{1}{2}\left[y_{1}, \partial_{+} y_{2}\right]_{G}^{i}+\frac{1}{2}\left[y_{2}, \partial_{+} y_{1}\right]_{G}^{i}-\left[y_{1}, \partial_{+} y_{2}\right]_{\tilde{G}}^{i}-\left[y_{2}, \partial_{+} y_{1}\right]_{\tilde{G}}^{i}-\frac{1}{3}\left[y_{1},\left[y_{1}, \partial_{+} y_{1}\right]_{G}\right]_{G}^{i}$

$+\frac{1}{2}\left[y_{1},\left[y_{1}, \partial_{+} y_{1}\right]_{G}\right]_{\tilde{G}}^{i}-\frac{1}{2}\left[\int_{0}^{\sigma^{+}}\left[y_{1}, \partial_{+} y_{1}\right]_{G} d \sigma^{\prime+}, \partial_{+} y_{1}\right]_{G}^{i}+\frac{1}{2}\left[\int_{0}^{\sigma^{+}}\left[y_{1}, \partial_{+} y_{1}\right]_{G} d \sigma^{\prime+}, \partial_{+} y_{1}\right]_{\tilde{G}}^{i}$ . . .

where we used subindex $G(\tilde{G})$ to represent commutation relations for the sigma model based on Lie group $G(\tilde{G})$. (1.i) gives $\tilde{y}_{L 1}=y_{L 1}+C_{L 1}$, where $C_{L 1}$ is a constant, and we set it equal to zero, and leads to (1.ii). Likewise second pseudoduality equation (5) gives the following infinite set of equations

$$
\begin{aligned}
& (2 . i) \partial_{-} \tilde{y}_{R 1}^{i}=-\partial_{-} y_{R 1}^{i} \\
& (2 . i i) \partial_{-} \tilde{y}_{R 2}^{i}-\frac{1}{2}\left[\tilde{y}_{R 1}, \partial_{-} \tilde{y}_{R 1}\right]_{\tilde{G}}^{i}=-\partial_{-} y_{R 2}^{i}+\frac{1}{2}\left[y_{R 1}, \partial_{-} y_{R 1}\right]_{G}^{i} \\
& (2 . i i i) \cdot \ldots
\end{aligned}
$$

where we used (2.i) and (1.i) in (2.ii), and (2.i) leads to $\tilde{y}_{R 1}=-y_{R 1}+C_{R 1}$, $C_{R 1}$ is a constant which is set to zero. We notice the fact that (22) only depends on $\sigma^{+}$, and (23) on $\sigma^{-}$point out pseudodual conserved currents, which can be written as follows

$$
\begin{gathered}
\tilde{J}_{+}^{L}\left(\sigma^{+}\right)=\tilde{g}^{-1} \partial_{+} \tilde{g}=\sum_{n=1}^{\infty} \varepsilon^{n} \tilde{J}_{+}^{L[n]}\left(\sigma^{+}\right) \\
\tilde{J}_{-}^{R}\left(\sigma^{-}\right)=\left(\partial_{-} \tilde{g}\right) \tilde{g}^{-1}=\sum_{n=1}^{\infty} \varepsilon^{n} \tilde{J}_{-}^{R[n]}\left(\sigma^{-}\right)
\end{gathered}
$$

where each component is determined by the orders of $\varepsilon$ 's, which are given by expression (20) (with tilde). The nonlocal expressions of currents are determined with the help of (22) and (23)

$$
\tilde{J}_{+}^{L[1]}\left(\sigma^{+}\right)=\partial_{+} \tilde{y}_{L 1}^{i}=\partial_{+} y_{L 1}^{i}
$$




$$
\begin{gathered}
\tilde{J}_{+}^{L[2]}\left(\sigma^{+}\right)=\partial_{+} \tilde{y}_{L 2}-\frac{1}{2}\left[\tilde{y}_{L 1}, \partial_{+} \tilde{y}_{L 1}\right]_{\tilde{G}}=\partial_{+} y_{L 2}^{i}+\frac{1}{2}\left[y_{L 1}, \partial_{+} y_{L 1}\right]_{G}^{i}-\left[y_{L 1}, \partial_{+} y_{L 1}\right]_{\tilde{G}} \\
\cdots \\
\tilde{J}_{-}^{R[1]}\left(\sigma^{-}\right)=\partial_{-} \tilde{y}_{R 1}^{i}=-\partial_{-} y_{R 1}^{i} \\
\tilde{J}_{-}^{R[2]}\left(\sigma^{-}\right)=\partial_{-} \tilde{y}_{R 2}+\frac{1}{2}\left[\tilde{y}_{R 1}, \partial_{-} \tilde{y}_{R 1}\right]_{\tilde{G}}=-\partial_{-} y_{R 2}^{i}+\frac{1}{2}\left[y_{R 1}, \partial_{-} y_{R 1}\right]_{G}^{i}+\left[y_{R 1}, \partial_{-} y_{R 1}\right]_{\tilde{G}}
\end{gathered}
$$

We see that these currents are conserved, $\partial_{-} \tilde{J}_{+}^{L}=\partial_{+} \tilde{J}_{-}^{R}=0$. It is observed that pseudodual currents are expressed as a nonlocal function of lie algebra valued fields on $\mathbf{g}$. As a result we obtained a family of nonlocal conserved currents on the WZW model on $G$. This family is a consequence of infinite set of terms of $T$ which is a function of lie algebra valued fields $\mathbf{g}$.

\subsection{An Example}

We consider sigma models based on Lie groups $G=S O(n+1)$ and $\tilde{G}=$ $S O(n, 1)$. The corresponding lie algebra are given by

$$
\mathbf{s o}(\mathbf{n}+\mathbf{1})=\left(\begin{array}{cc}
a & b \\
-b^{t} & c
\end{array}\right) \quad \mathbf{s o}(\mathbf{n}, \mathbf{1})=\left(\begin{array}{cc}
\tilde{a} & \tilde{b} \\
\tilde{b}^{t} & \tilde{c}
\end{array}\right) \quad \begin{aligned}
& a=\tilde{a}=n \times n \\
& b=\tilde{b}=n \times 1 \\
& c=\tilde{c}=1 \times 1
\end{aligned}
$$

Let $g=e^{Y}$ and $\tilde{g}=e^{\tilde{Y}}$, and fields $g_{L}^{-1} \partial_{+} g_{L}$ and $\tilde{g}_{L}^{-1} \partial_{+} \tilde{g_{L}}$ are given by (14). We get the following expressions

$$
\begin{aligned}
& Y_{L}=\left(\begin{array}{cc}
a_{L} & b_{L} \\
-b_{L}^{t} & c_{L}
\end{array}\right) \quad \partial_{+} Y_{L}=\left(\begin{array}{cc}
\partial_{+} a_{L} & \partial_{+} b_{L} \\
-\partial_{+} b_{L}^{t} & \partial_{+} c_{L}
\end{array}\right) \\
& \tilde{Y}_{L}=\left(\begin{array}{cc}
\tilde{a_{L}} & \tilde{b_{L}} \\
\tilde{b_{L}} & \tilde{c_{L}}
\end{array}\right) \quad \partial_{+} \tilde{Y}_{L}=\left(\begin{array}{cc}
\partial_{+} \tilde{a_{L}} & \partial_{+} \tilde{b_{L}} \\
\partial_{+} \tilde{b_{L}} & \tilde{\partial_{+}} \tilde{c_{L}}
\end{array}\right) \\
& {\left[Y_{L}, \partial_{+} Y_{L}\right]=\left(\begin{array}{cc}
0 & b_{L}^{t}\left(\partial_{+} a_{L}\right)-c_{L}\left(\partial_{+} b_{L}^{t}\right)+\left(\partial_{+} b_{L}^{t}\right) a_{L}+\left(\partial_{+} c_{L}\right) b_{L}^{t} \\
a_{L} \partial_{+} b_{L}+b_{L} \partial_{+} c_{L}-\left(\partial_{+} a_{L}\right) b_{L}-\left(\partial_{+} b_{L}\right) c_{L}
\end{array}\right)} \\
& {\left[\tilde{Y}_{L}, \partial_{+} \tilde{Y}_{L}\right]=\left(\begin{array}{cc}
0 & \tilde{b}_{L}^{t}\left(\partial_{+} \tilde{a}_{L}\right)+\tilde{c}_{L}\left(\partial_{+} \tilde{b}_{L}^{t}\right)-\left(\partial_{+} \tilde{b}_{L}^{t}\right) \tilde{a}_{L}-\left(\partial_{+} \tilde{c}_{L}\right) \tilde{b}_{L}^{t} \\
\tilde{a}_{L} \partial_{+} \tilde{b}_{L}+\tilde{b}_{L} \partial_{+} \tilde{c}_{L}-\left(\partial_{+} \tilde{a}_{L}\right) \tilde{b}_{L}-\left(\partial_{+} \tilde{b}_{L}\right) \tilde{c}_{L}
\end{array}\right)}
\end{aligned}
$$


Hence up to the second order terms we get the expressions for the fields on the target space elements

$$
g_{L}^{-1} \partial_{+} g_{L}=\left(\begin{array}{ll}
X_{1} & X_{2} \\
X_{3} & X_{4}
\end{array}\right)+H . O \quad \tilde{g}_{L}^{-1} \partial_{+} \tilde{g_{L}}=\left(\begin{array}{cc}
\tilde{X}_{1} & \tilde{X}_{2} \\
\tilde{X}_{3} & \tilde{X}_{4}
\end{array}\right)+H . O
$$

where we defined the following

$$
\begin{gathered}
X_{1}=\partial_{+} a_{L} \quad \tilde{X}_{1}=\partial_{+} \tilde{a}_{L} \quad X_{4}=\partial_{+} c_{L} \quad \tilde{X}_{4}=\partial_{+} \tilde{c}_{L} \\
X_{2}=\partial_{+} b_{L}-\frac{a_{L} \partial_{+} b_{L}+b_{L} \partial_{+} c_{L}-\left(\partial_{+} a_{L}\right) b_{L}-\left(\partial_{+} b_{L}\right) c_{L}}{2} \\
\tilde{X}_{2}=\partial_{+} \tilde{b}_{L}-\frac{\tilde{a}_{L} \partial_{+} \tilde{b}_{L}+\tilde{b}_{L} \partial_{+} \tilde{c}_{L}-\left(\partial_{+} \tilde{a}_{L}\right) \tilde{b}_{L}-\left(\partial_{+} \tilde{b}_{L}\right) \tilde{c}_{L}}{2} \\
X_{3}=-\partial_{+} b_{L}^{t}-\frac{-b_{L}^{t}\left(\partial_{+} a_{L}\right)-c_{L}\left(\partial_{+} b_{L}^{t}\right)+\left(\partial_{+} b_{L}^{t}\right) a_{L}+\left(\partial_{+} c_{L}\right) b_{L}^{t}}{2} \\
\tilde{X}_{3}=\partial_{+} \tilde{b}_{L}^{t}-\frac{\tilde{b}_{L}^{t}\left(\partial_{+} \tilde{a}_{L}\right)+\tilde{c}_{L}\left(\partial_{+} \tilde{b}_{L}^{t}\right)-\left(\partial_{+} \tilde{b}_{L}^{t}\right) \tilde{a}_{L}-\left(\partial_{+} \tilde{c}_{L}\right) \tilde{b}_{L}^{t}}{2}
\end{gathered}
$$

Likewise we get the following expressions related to fields $g^{-1} \partial_{-} g$ and $\tilde{g}^{-1} \partial_{-} \tilde{g}$ using (15)

$$
\begin{aligned}
& {\left[Y_{L}, \partial_{-} Y_{R}\right]=\left(\begin{array}{cc}
0 & a_{L} \partial_{-} b_{R}+b_{L} \partial_{-} c_{R}-\left(\partial_{-} a_{R}\right) b_{L}-\left(\partial_{-} b_{R}\right) c_{L} \\
-b_{L}^{t}\left(\partial_{-} a_{R}\right)-c_{L}\left(\partial_{-} b_{R}^{t}\right)+\left(\partial_{-} b_{R}^{t}\right) a_{L}+\left(\partial_{-} c_{R}\right) b_{L}^{t} & 0
\end{array}\right)} \\
& {\left[Y_{R}, \partial_{-} Y_{R}\right]=\left(\begin{array}{cc}
0 & a_{R} \partial_{-} b_{R}+b_{R} \partial_{-} c_{R}-\left(\partial_{-} a_{R}\right) b_{R}-\left(\partial_{-} b_{R}\right) c_{R} \\
-b_{R}^{t}\left(\partial_{-} a_{R}\right)-c_{R}\left(\partial_{-} b_{R}^{t}\right)+\left(\partial_{-} b_{R}^{t}\right) a_{R}+\left(\partial_{-} c_{R}\right) b_{R}^{t} & 0
\end{array}\right)} \\
& {\left[\tilde{Y}_{L}, \partial_{-} \tilde{Y}_{R}\right]=\left(\begin{array}{cc}
0 & \tilde{b}_{L}^{t}\left(\partial_{-} \tilde{a}_{R}\right)+\tilde{c}_{L}\left(\partial_{-} \tilde{b}_{R}^{t}\right)-\left(\partial_{-} \tilde{b}_{R}^{t}\right) \tilde{a}_{L}-\left(\partial_{-} \tilde{c}_{R}\right) \tilde{b}_{L}^{t} \\
\tilde{a}_{L} \partial_{-} \tilde{b}_{R}+\tilde{b}_{L} \partial_{-} \tilde{c}_{R}-\left(\partial_{-} \tilde{a}_{R}\right) \tilde{b}_{L}-\left(\partial_{-} \tilde{b}_{R}\right) \tilde{c}_{L}
\end{array}\right)} \\
& {\left[\tilde{Y}_{R}, \partial_{-} \tilde{Y}_{R}\right]=\left(\begin{array}{cc}
0 & \tilde{a}_{R} \partial_{-} \tilde{b}_{R}+\tilde{b}_{R} \partial_{-} \tilde{c}_{R}-\left(\partial_{-} \tilde{a}_{R}\right) \tilde{b}_{R}-\left(\partial_{-} \tilde{b}_{R}\right) \tilde{c}_{R} \\
\tilde{b}_{R}^{t}\left(\partial_{-} \tilde{a}_{R}\right)+\tilde{c}_{R}\left(\partial_{-} \tilde{b}_{R}^{t}\right)-\left(\partial_{-} \tilde{b}_{R}^{t}\right) \tilde{a}_{R}-\left(\partial_{-} \tilde{c}_{R}\right) \tilde{b}_{R}^{t} & 0
\end{array}\right)} \\
& g^{-1} \partial_{-} g=\left(\begin{array}{cc}
Z_{1} & Z_{2} \\
Z_{3} & Z_{4}
\end{array}\right)+H . O \quad \tilde{g}^{-1} \partial_{-} \tilde{g}=\left(\begin{array}{cc}
\tilde{Z}_{1} & \tilde{Z}_{2} \\
\tilde{Z}_{3} & \tilde{Z}_{4}
\end{array}\right)+H . O \\
& Z_{1}=\partial_{-} a_{R} \quad \tilde{Z}_{1}=\partial_{-} \tilde{a}_{R} \quad Z_{4}=\partial_{-} c_{R} \quad \tilde{Z}_{4}=\partial_{-} \tilde{c}_{R} \\
& Z_{2}=\partial_{-} b_{R}-\left(a_{L}+\frac{a_{R}}{2}\right) \partial_{-} b_{R}-\left(b_{L}+\frac{b_{R}}{2}\right) \partial_{-} c_{R}+\left(\partial_{-} a_{R}\right)\left(b_{L}+\frac{b_{R}}{2}\right)+\left(\partial_{-} b_{R}\right)\left(c_{L}+\frac{c_{R}}{2}\right) \\
& \tilde{Z}_{2}=\partial_{-} \tilde{b}_{R}-\left(\tilde{a}_{L}+\frac{\tilde{a}_{R}}{2}\right) \partial_{-} \tilde{b}_{R}-\left(\tilde{b}_{L}+\frac{\tilde{b}_{R}}{2}\right) \partial_{-} \tilde{c}_{R}+\left(\partial_{-} \tilde{a}_{R}\right)\left(\tilde{b}_{L}+\frac{\tilde{b}_{R}}{2}\right)+\left(\partial_{-} \tilde{b}_{R}\right)\left(\tilde{c}_{L}+\frac{\tilde{c}_{R}}{2}\right)
\end{aligned}
$$




$$
\begin{aligned}
& Z_{3}=-\partial_{-} b_{R}^{t}+\left(b_{L}^{t}+\frac{b_{R}^{t}}{2}\right) \partial_{-} a_{R}+\left(c_{L}+\frac{c_{R}}{2}\right) \partial_{-} b_{R}^{t}-\left(\partial_{-} b_{R}^{t}\right)\left(a_{L}+\frac{a_{R}}{2}\right)-\left(\partial_{-} c_{R}\right)\left(b_{L}^{t}+\frac{b_{R}^{t}}{2}\right) \\
& \tilde{Z}_{3}=\partial_{-} \tilde{b}_{R}^{t}-\left(\tilde{b}_{L}^{t}+\frac{\tilde{b}_{R}^{t}}{2}\right) \partial_{-} \tilde{a}_{R}-\left(\tilde{c}_{L}+\frac{\tilde{c}_{R}}{2}\right) \partial_{-} \tilde{b}_{R}^{t}+\left(\partial_{-} \tilde{b}_{R}^{t}\right)\left(\tilde{a}_{L}+\frac{\tilde{a}_{R}}{2}\right)+\left(\partial_{-} \tilde{c}_{R}\right)\left(\tilde{b}_{L}^{t}+\frac{\tilde{b}_{R}^{t}}{2}\right)
\end{aligned}
$$

Obviously equations of motion are satisfied. Since we want to reduce constraints on the conservation laws and bring the nonlinear characters of conserved currents into the open we let $e=\sum_{n=1}^{\infty} \varepsilon^{n} e_{n}$, where e stands for the matrix components $a, b$ and $c$. We may find solutions in the orders of $\varepsilon$ 's. But we need to find transformation matrix $T$ first and foremost.

\subsubsection{Trivial Case: $\mathrm{T}=\mathrm{I}$}

Let us consider first a trivial solution where transformation matrix is identity. Pseudoduality equations will be

$$
\begin{aligned}
\left({\tilde{g_{L}}}^{-1} \partial_{+} \tilde{g_{L}}\right)^{i} & =\left(g_{L}^{-1} \partial_{+} g_{L}\right)^{i} \\
\left(\tilde{g}^{-1} \partial_{-} \tilde{g}\right)^{i} & =-\left(g^{-1} \partial_{-} g\right)^{i}
\end{aligned}
$$

Using (31) the first equation (33) leads to

$$
\begin{array}{cc}
\partial_{+} \tilde{a}_{L 1}=\partial_{+} a_{L 1} & \partial_{+} \tilde{a}_{L 2}=\partial_{+} a_{L 2} \\
\partial_{+} \tilde{c}_{L 1}=\partial_{+} c_{L 1} & \partial_{+} \tilde{c}_{L 2}=\partial_{+} c_{L 2} \\
\partial_{+} \tilde{b}_{L 1}=\partial_{+} b_{L 1} & \partial_{+} \tilde{b}_{L 1}^{t}=-\partial_{+} b_{L 1}^{t} \\
\partial_{+} \tilde{b_{L 2}}=\partial_{+} b_{L 2}+\frac{1}{2}\left[A_{L 1}\left(\partial_{+} b_{L 1}\right)+B_{L 1}\left(\partial_{+} c_{L 1}\right)-\left(\partial_{+} a_{L 1}\right) B_{L 1}-\left(\partial_{+} b_{L 1}\right) C_{L 1}\right] \\
\partial_{+} \tilde{b}_{L 2}^{t}=-\partial_{+} b_{L 2}^{t}-\frac{1}{2}\left[B_{L 1}^{t}\left(\partial_{+} a_{L 1}\right)+C_{L 1}\left(\partial_{+} b_{L 1}^{t}\right)-\left(\partial_{+} b_{L 1}^{t}\right) A_{L 1}-\left(\partial_{+} c_{L 1}\right) B_{L 1}^{t}\right]
\end{array}
$$

where we used the solutions of first six equations in the last two lines as follows

$$
\begin{array}{cc}
\tilde{a}_{L 1}=a_{L 1}+A_{L 1} & \tilde{a}_{L 2}=a_{L 2}+A_{L 2} \\
\tilde{c}_{L 1}=c_{L 1}+C_{L 1} & \tilde{c}_{L 2}=c_{L 2}+C_{L 2} \\
\tilde{b}_{L 1}=b_{L 1}+B_{L 1} & \tilde{b}_{L 1}^{t}=-b_{L 1}^{t}-B_{L 1}^{t} \\
\tilde{b_{L 2}}=b_{L 2}+\frac{1}{2}\left(A_{L 1} b_{L 1}+B_{L 1} c_{L 1}-a_{L 1} B_{L 1}-b_{L 1} C_{L 1}\right)+B_{L 2} \\
\tilde{b}_{L 2}^{t}=-b_{L 2}^{t}-\frac{1}{2}\left(B_{L 1}^{t} a_{L 1}+C_{L 1}\left(\partial_{+} b_{L 1}^{t}\right)-\left(\partial_{+} b_{L 1}\right) A_{L 1}-c_{L 1} B_{L 1}^{t}\right)-B_{L 2}^{t}
\end{array}
$$

where $A_{L 1}, A_{L 2}, B_{L 1}, B_{L 2}, C_{L 1}$ and $C_{L 2}$ are constants. Therefore pseudodual left current (31) up to the order of $\varepsilon^{2}$ in nonlocal expressions is

$$
\tilde{g}_{L}^{-1} \partial_{+} \tilde{g}_{L}=\left(\begin{array}{cc}
\tilde{M}_{1} & \tilde{M}_{2} \\
\tilde{M}_{3} & \tilde{M}_{4}
\end{array}\right)+H . O
$$


where we defined the following symbols for the entries of matrix

$$
\begin{gathered}
\tilde{M}_{1}=\varepsilon \partial_{+} \tilde{a}_{L 1}+\varepsilon^{2} \partial_{+} \tilde{a}_{L 2}=\varepsilon \partial_{+} a_{L 1}+\varepsilon^{2} \partial_{+} a_{L 2} \\
\tilde{M}_{4}=\varepsilon \partial_{+} \tilde{c}_{L 1}+\varepsilon^{2} \partial_{+} \tilde{c}_{L 2}=\varepsilon \partial_{+} c_{L 1}+\varepsilon^{2} \partial_{+} c_{L 2} \\
\tilde{M}_{2}=\varepsilon \partial_{+} \tilde{b}_{L 1}+\varepsilon^{2}\left[\partial_{+} \tilde{b}_{L 2}-\frac{1}{2}\left(\tilde{a}_{L 1} \partial_{+} \tilde{b}_{L 1}+\tilde{b}_{L 1} \partial_{+} \tilde{c}_{L 1}-\left(\partial_{+} \tilde{a}_{L 1}\right) \tilde{b}_{L 1}-\left(\partial_{+} \tilde{b}_{L 1}\right) \tilde{c}_{L 1}\right)\right] \\
=\varepsilon \partial_{+} b_{L 1}+\varepsilon^{2}\left[\partial_{+} b_{L 2}-\frac{1}{2}\left[a_{L 1}\left(\partial_{+} b_{L 1}\right)+b_{L 1}\left(\partial_{+} c_{L 1}\right)-\left(\partial_{+} a_{L 1}\right) b_{L 1}-\left(\partial_{+} b_{L 1}\right) c_{L 1}\right]\right] \\
\tilde{M}_{3}=\varepsilon \partial_{+} \tilde{b}_{L 1}^{t}+\varepsilon^{2}\left[\partial_{+} \tilde{b}_{L 2}^{t}-\frac{1}{2}\left[\tilde{b}_{L 1}^{t}\left(\partial_{+} \tilde{a}_{L 1}\right)+\tilde{c}_{L 1}\left(\partial_{+} \tilde{b}_{L 1}^{t}\right)-\left(\partial_{+} \tilde{b}_{L 1}^{t}\right) \tilde{a}_{L 1}-\left(\partial_{+} \tilde{c}_{L 1}\right) \tilde{b}_{L 1}^{t}\right]\right] \\
=-\varepsilon \partial_{+} b_{L 1}^{t}-\varepsilon^{2}\left[\partial_{+} b_{L 2}^{t}-\frac{1}{2}\left[b_{L 1}^{t}\left(\partial_{+} a_{L 1}\right)+c_{L 1}\left(\partial_{+} b_{L 1}^{t}\right)-\left(\partial_{+} b_{L 1}^{t}\right) a_{L 1}-\left(\partial_{+} c_{L 1}\right) b_{L 1}^{t}\right]\right]
\end{gathered}
$$

Obviously this current is conserved. To find right current we use $2^{\text {nd }}$ pseudoduality equation (34) and we find the following expressions up to the order of $\varepsilon^{2}$

$$
\begin{aligned}
& \partial_{-} \tilde{a}_{R 1}=-\partial_{-} a_{R 1} \quad \partial_{-} \tilde{a}_{R 2}=-\partial_{-} a_{R 2} \\
& \partial_{-} \tilde{c}_{R 1}=-\partial_{-} c_{R 1} \quad \partial_{-} \tilde{c}_{R 2}=-\partial_{-} c_{R 2} \\
& \partial_{-} \tilde{b}_{R 1}=-\partial_{-} b_{R 1} \quad \partial_{-} \tilde{b}_{R 1}^{t}=\partial_{-} b_{R 1}^{t} \\
& \partial_{-} \tilde{b_{R 2}}=-\partial_{-} b_{R 2}+\left(a_{R 1}-A_{L 1}+\frac{A_{R 1}}{2}\right)\left(\partial_{-} b_{R 1}\right)+\left(b_{R 1}-B_{L 1}+\frac{B_{R 1}}{2}\right)\left(\partial_{-} c_{R 1}\right) \\
& -\left(\partial_{-} a_{R 1}\right)\left(b_{R 1}-B_{L 1}+\frac{B_{R 1}^{2}}{2}\right)-\left(\partial_{-} b_{R 1}\right)\left(c_{R 1}-C_{L 1}+\frac{C_{R 1}^{2}}{2}\right) \\
& \partial_{-} \tilde{b}_{R 2}^{t}=\partial_{-} b_{R 2}^{t}-\left(-B_{L 1}^{t}+b_{R 1}^{t}+\frac{B_{R 1}^{t}}{2}\right)\left(\partial_{-} a_{R 1}\right)-\left(-C_{L 1}+c_{R 1}+\frac{C_{R 1}}{2}\right)\left(\partial_{-} b_{R 1}^{t}\right) \\
& +\left(\partial_{-} b_{R 1}^{t}\right)\left(-A_{L 1}+a_{R 1}+\frac{A_{R 1}}{2}\right)+\left(\partial_{-} c_{R 1}\right)\left(-B_{L 1}^{t}+b_{R 1}^{t}+\frac{B_{R 1}^{t}}{2}\right)
\end{aligned}
$$

where we used the solution of first six equations in the last two equations as

$$
\begin{array}{cl}
\tilde{a}_{R 1}=-a_{R 1}-A_{R 1} & \tilde{a}_{R 2}=-a_{R 2}-A_{R 2} \\
\tilde{c}_{R 1}=-c_{R 1}-C_{R 1} & \tilde{c}_{R 2}=-c_{R 2}-C_{R 2} \\
\tilde{b}_{R 1}=-b_{R 1}-B_{R 1} & \tilde{b}_{R 1}^{t}=b_{R 1}^{t}+B_{R 1}^{t}
\end{array}
$$

where $A_{R 1}, A_{R 2}, B_{R 1}, C_{R 1}$ and $C_{R 2}$ are constants. A brief computation yields the following expression for the right current

$$
\left(\partial_{-} \tilde{g}_{R}\right) \tilde{g}_{R}^{-1}=\left(\begin{array}{cc}
\tilde{N}_{1} & \tilde{N}_{2} \\
\tilde{N}_{3} & \tilde{N}_{4}
\end{array}\right)+H . O
$$




$$
\begin{gathered}
\tilde{N}_{1}=\varepsilon \partial_{-} \tilde{a}_{R 1}+\varepsilon^{2} \partial_{-} \tilde{a}_{R 2}=-\varepsilon \partial_{-} a_{R 1}-\varepsilon^{2} \partial_{-} a_{R 2} \\
\tilde{N}_{4}=\varepsilon \partial_{-} \tilde{c}_{R 1}+\varepsilon^{2} \partial_{-} \tilde{c}_{R 2}=-\varepsilon \partial_{-} c_{R 1}-\varepsilon^{2} \partial_{-} c_{R 2} \\
\tilde{N}_{2}=\varepsilon \partial_{-} \tilde{b}_{R 1}+\varepsilon^{2}\left[\partial_{-} \tilde{b}_{R 2}+\frac{1}{2}\left(\tilde{a}_{R 1} \partial_{-} \tilde{b}_{R 1}+\tilde{b}_{R 1} \partial_{-} \tilde{c}_{R 1}-\left(\partial_{-} \tilde{a}_{R 1}\right) \tilde{b}_{R 1}-\left(\partial_{-} \tilde{b}_{R 1}\right) \tilde{c}_{R 1}\right)\right] \\
=-\varepsilon \partial_{-} b_{R 1}+\varepsilon^{2}\left[-\partial_{-} b_{R 2}+\left(\frac{3}{2} a_{R 1}+A_{R 1}-A_{L 1}\right)\left(\partial_{-} b_{R 1}\right)+\left(\frac{3}{2} b_{R 1}+B_{R 1}-B_{L 1}\right)\left(\partial_{-} c_{R 1}\right)\right. \\
\left.-\left(\partial_{-} a_{R 1}\right)\left(\frac{3}{2} b_{R 1}+B_{R 1}-B_{L 1}\right)-\left(\partial_{-} b_{R 1}\right)\left(\frac{3}{2} c_{R 1}+C_{R 1}-C_{L 1}\right)\right] \\
\tilde{N}_{3}=\varepsilon \partial_{-} \tilde{b}_{R 1}^{t}+\varepsilon^{2}\left[\partial_{-} \tilde{b}_{R 2}^{t}+\frac{1}{2}\left[\tilde{b}_{R 1}^{t}\left(\partial_{-} \tilde{a}_{R 1}\right)+\tilde{c}_{R 1}\left(\partial_{-} \tilde{b}_{R 1}^{t}\right)-\left(\partial_{-} \tilde{b}_{R 1}^{t}\right) \tilde{a}_{R 1}-\left(\partial_{-} \tilde{c}_{R 1}\right) \tilde{b}_{R 1}^{t}\right]\right] \\
=\varepsilon \partial_{-} b_{R 1}^{t}+\varepsilon^{2}\left[\partial_{-} b_{R 2}^{t}-\left(\frac{3}{2} b_{R 1}^{t}+B_{R 1}^{t}-B_{L 1}^{t}\right)\left(\partial_{-} a_{R 1}\right)-\left(\frac{3}{2} c_{R 1}+C_{R 1}-C_{L 1}\right)\left(\partial_{-} b_{R 1}^{t}\right)\right. \\
\left.+\left(\partial_{-} b_{R 1}^{t}\right)\left(\frac{3}{2} a_{R 1}+A_{R 1}-A_{L 1}\right)+\left(\partial_{-} c_{R 1}\right)\left(\frac{3}{2} b_{R 1}^{t}+B_{R 1}^{t}-B_{L 1}^{t}\right)\right]
\end{gathered}
$$

We see that this current is also conserved.

\subsubsection{Nontrivial Case: General T}

In this case we use the general expression (19) of transformation matrix $\mathrm{T}$. Pseudoduality equations are given by (4) and (5), and gave us the equations (22) and (23) which can be written as

$$
\begin{aligned}
& \partial_{+} \tilde{a}_{L 1}=\partial_{+} a_{L 1} \quad \partial_{+} \tilde{b}_{L 1}=\partial_{+} b_{L 1} \quad \partial_{+} \tilde{b}_{L 1}^{t}=-\partial_{+} b_{L 1}^{t} \quad \partial_{+} \tilde{c}_{L 1}=\partial_{+} c_{L 1} \\
& \partial_{-} \tilde{a}_{R 1}=-\partial_{-} a_{R 1} \quad \partial_{-} \tilde{b}_{R 1}=-\partial_{-} b_{R 1} \quad \partial_{-} \tilde{b}_{R 1}^{t}=\partial_{-} b_{R 1}^{t} \quad \partial_{-} \tilde{c}_{R 1}=-\partial_{-} c_{R 1} \\
& \partial_{+} \tilde{a}_{L 2}=\partial_{+} a_{L 2} \quad \partial_{+} \tilde{c}_{L 2}=\partial_{+} c_{L 2} \quad \partial_{-} \tilde{a}_{R 2}=-\partial_{-} a_{R 2} \quad \partial_{-} \tilde{c}_{R 2}=-\partial_{-} c_{R 2} \\
& \partial_{+} \tilde{b}_{L 2}=\partial_{+} b_{L 2}-\frac{1}{2}\left[A_{L 1}\left(\partial_{+} b_{L 1}\right)+B_{L 1}\left(\partial_{+} c_{L 1}\right)-\left(\partial_{+} a_{L 1}\right) B_{L 1}-\left(\partial_{+} b_{L 1}\right) C_{L 1}\right] \\
& \partial_{+} \tilde{b}_{L 2}^{t}=-\partial_{+} b_{L 2}^{t}+\frac{1}{2}\left[B_{L 1}^{t}\left(\partial_{+} a_{L 1}\right)+C_{L 1}\left(\partial_{+} b_{L 1}^{t}\right)-\left(\partial_{+} b_{L 1}^{t}\right) A_{L 1}-\left(\partial_{+} c_{L 1}\right) B_{L 1}^{t}\right] \\
& \partial_{-} \tilde{b}_{R 2}=-\partial_{-} b_{R 2}+\left(a_{R 1}+\frac{A_{R 1}}{2}\right)\left(\partial_{-} b_{R 1}\right)+\left(b_{R 1}+\frac{B_{R 1}}{2}\right)\left(\partial_{-} c_{R 1}\right) \\
& -\left(\partial_{-} a_{R 1}\right)\left(b_{R 1}+\frac{B_{R 1}}{2}\right)-\left(\partial_{-} b_{R 1}\right)\left(c_{R 1}+\frac{C_{R 1}}{2}\right) \\
& \partial_{-} \tilde{b}_{R 2}^{t}=\partial_{-} b_{R 2}^{t}-\left(b_{R 1}^{t}+\frac{B_{R 1}^{t}}{2}\right)\left(\partial_{-} a_{R 1}\right)-\left(c_{R 1}+\frac{C_{R 1}}{2}\right)\left(\partial_{-} b_{R 1}^{t}\right) \\
& +\left(\partial_{-} b_{R 1}^{t}\right)\left(a_{R 1}+\frac{A_{R 1}}{2}\right)+\left(\partial_{-} c_{R 1}\right)\left(b_{R 1}^{t}+\frac{B_{R 1}^{t}}{2}\right)
\end{aligned}
$$


where we used the solutions of first three lines for the last four expressions. Solutions of these equations are

$$
\begin{array}{ccc}
\tilde{a}_{L 1}=a_{L 1}+A_{L 1} & \tilde{b}_{L 1}=b_{L 1}+B_{L 1} & \tilde{b}_{L 1}^{t}=-b_{L 1}^{t}-B_{L 1}^{t} \\
\tilde{c}_{L 1}=c_{L 1}+C_{L 1} & \tilde{a}_{R 1}=-a_{R 1}-A_{R 1} & \tilde{b}_{R 1}=-b_{R 1}-B_{R 1} \\
\tilde{b}_{R 1}^{t}=b_{R 1}^{t}+B_{R 1}^{t} & \tilde{c}_{R 1}=-c_{R 1}-C_{R 1} & \tilde{a}_{L 2}=a_{L 2}+A_{L 2} \\
\tilde{c}_{L 2}=c_{L 2}+C_{L 2} & \tilde{a}_{R 2}=-a_{R 2}-A_{R 2} & \tilde{c}_{R 2}=-c_{R 2}-C_{R 2} \\
\tilde{b}_{L 2}=b_{L 2}+B_{L 2}-\frac{1}{2}\left[A_{L 1} b_{L 1}+B_{L 1} c_{L 1}-a_{L 1} B_{L 1}-b_{L 1} C_{L 1}\right] \\
\tilde{b}_{L 2}^{t}=-b_{L 2}^{t}-B_{L 2}^{t}+\frac{1}{2}\left[B_{L 1}^{t} a_{L 1}+C_{L 1} b_{L 1}^{t}-b_{L 1}^{t} A_{L 1}-c_{L 1} B_{L 1}^{t}\right]
\end{array}
$$

where $A_{L 1}, A_{R 1}, B_{L 1}, B_{R 1}, C_{L 1}, C_{R 1}$, and $B_{L 2}$ are constants. We did not find solutions of $\tilde{b}_{R 2}$ and $\tilde{b}_{R 2}^{t}$ because of their complicated forms and no need to use them. Hence pseudodual left current (24) will be

$$
\begin{aligned}
\tilde{J}_{+}^{(L)} & =\tilde{g}^{-1} \partial_{+} \tilde{g}=\varepsilon \partial_{+} \tilde{y}_{L 1}+\varepsilon^{2}\left\{\partial_{+} \tilde{y}_{L 2}-\frac{1}{2}\left[\tilde{y}_{L 1}, \partial_{+} \tilde{y}_{L 1}\right]_{\tilde{G}}\right\}+H . O . \\
& =\left(\begin{array}{cc}
\tilde{M}_{1} & \tilde{M}_{2} \\
\tilde{M}_{3} & \tilde{M}_{4}
\end{array}\right)+H . O .
\end{aligned}
$$

where

$$
\begin{aligned}
\tilde{M}_{1} & =\varepsilon \partial_{+} \tilde{a}_{L 1}+\varepsilon^{2} \partial_{+} \tilde{a}_{L 2}=\varepsilon \partial_{+} a_{L 1}+\varepsilon^{2} \partial_{+} a_{L 2} \\
\tilde{M}_{4} & =\varepsilon \partial_{+} \tilde{c}_{L 1}+\varepsilon^{2} \partial_{+} \tilde{c}_{L 2}=\varepsilon \partial_{+} c_{L 1}+\varepsilon^{2} \partial_{+} c_{L 2} \\
\tilde{M}_{2} & =\varepsilon \partial_{+} \tilde{b}_{L 1}+\varepsilon^{2}\left[\partial_{+} \tilde{b}_{L 2}-\frac{1}{2}\left\{\tilde{a}_{L 1}\left(\partial_{+} \tilde{b}_{L 1}\right)+\tilde{b}_{L 1}\left(\partial_{+} \tilde{c}_{L 1}\right)-\left(\partial_{+} \tilde{a}_{L 1}\right) \tilde{b}_{L 1}-\left(\partial_{+} \tilde{b}_{L 1}\right) \tilde{c}_{L 1}\right\}\right] \\
& =\varepsilon \partial_{+} b_{L 1}+\varepsilon^{2}\left[\partial_{+} b_{L 2}-\frac{1}{2}\left\{a_{L 1}\left(\partial_{+} b_{L 1}\right)+b_{L 1}\left(\partial_{+} c_{L 1}\right)-\left(\partial_{+} a_{L 1}\right) b_{L 1}-\left(\partial_{+} b_{L 1}\right) c_{L 1}\right\}\right] \\
\tilde{M}_{3} & =\varepsilon \partial_{+} \tilde{b}_{L 1}^{t}+\varepsilon^{2}\left[\partial_{+} \tilde{b}_{L 2}^{t}-\frac{1}{2}\left\{\tilde{b}_{L 1}^{t}\left(\partial_{+} \tilde{a}_{L 1}\right)+\tilde{c}_{L 1}\left(\partial_{+} \tilde{b}_{L 1}^{t}\right)-\left(\partial_{+} \tilde{b}_{L 1}^{t}\right) \tilde{a}_{L 1}-\left(\partial_{+} \tilde{c}_{L 1}\right) \tilde{b}_{L 1}^{t}\right\}\right] \\
& =-\varepsilon \partial_{+} b_{L 1}^{t}-\varepsilon^{2}\left[\partial_{+} b_{L 2}^{t}-\left(\frac{b_{L 1}^{t}}{2}+B_{L 1}^{t}\right)\left(\partial_{+} a_{L 1}\right)-\left(\frac{c_{L 1}}{2}+C_{L 1}\right)\left(\partial_{+} b_{L 1}^{t}\right)\right. \\
& \left.+\left(\partial_{+} b_{L 1}^{t}\right)\left(\frac{a_{L 1}}{2}+A_{L 1}\right)+\left(\partial_{+} c_{L 1}\right)\left(\frac{b_{L 1}^{t}}{2}+B_{L 1}^{t}\right)\right]
\end{aligned}
$$


Pseudodual right current (25) can be constructed as follows

$$
\begin{aligned}
\tilde{J}_{-}^{(R)} & =\left(\partial_{-} \tilde{g}\right) \tilde{g}^{-1}=\varepsilon \partial_{-} \tilde{y}_{R 1}+\varepsilon^{2}\left\{\partial_{-} \tilde{y}_{R 2}+\frac{1}{2}\left[\tilde{y}_{R 1}, \partial_{-} \tilde{y}_{R 1}\right]_{\tilde{G}}\right\}+\text { H.O. } \\
& =\left(\begin{array}{cc}
\tilde{N}_{1} & \tilde{N}_{2} \\
\tilde{N}_{3} & \tilde{N}_{4}
\end{array}\right)+\text { H.O. }
\end{aligned}
$$

where

$$
\begin{aligned}
\tilde{N}_{1} & =\varepsilon \partial_{-} \tilde{a}_{R 1}+\varepsilon^{2} \partial_{-} \tilde{a}_{R 2}=-\varepsilon \partial_{-} a_{R 1}-\varepsilon^{2} \partial_{-} a_{R 2} \\
\tilde{N}_{4} & =\varepsilon \partial_{-} \tilde{c}_{R 1}+\varepsilon^{2} \partial_{-} \tilde{c}_{R 2}=-\varepsilon \partial_{-} c_{R 1}-\varepsilon^{2} \partial_{-} c_{R 2} \\
\tilde{N}_{2} & =\varepsilon \partial_{-} \tilde{b}_{R 1}+\varepsilon^{2}\left\{\partial_{-} \tilde{b}_{R 2}+\frac{1}{2}\left[\tilde{a}_{R 1}\left(\partial_{-} \tilde{b}_{R 1}\right)+\tilde{b}_{R 1}\left(\partial_{-} \tilde{c}_{R 1}\right)-\left(\partial_{-} \tilde{a}_{R 1}\right) \tilde{b}_{R 1}-\left(\partial_{-} \tilde{b}_{R 1}\right) \tilde{c}_{R 1}\right]\right\} \\
& =-\varepsilon \partial_{-} b_{R 1}-\varepsilon^{2}\left\{\partial_{-} b_{R 2}-\left(\frac{3 a_{R 1}}{2}+A_{R 1}\right)\left(\partial_{-} b_{R 1}\right)-\left(\frac{3 b_{R 1}}{2}+B_{R 1}\right)\left(\partial_{-} c_{R 1}\right)\right. \\
& \left.+\left(\partial_{-} a_{R 1}\right)\left(\frac{3 b_{R 1}}{2}+B_{R 1}\right)+\left(\partial_{-} b_{R 1}\right)\left(\frac{3 c_{R 1}}{2}+C_{R 1}\right)\right\} \\
\tilde{N}_{3} & =\varepsilon \partial_{-} \tilde{b}_{R 1}^{t}+\varepsilon^{2}\left\{\partial_{-} \tilde{b}_{R 2}^{t}+\frac{1}{2}\left[\tilde{b}_{R 1}^{t}\left(\partial_{-} \tilde{a}_{R 1}\right)+\tilde{c}_{R 1}\left(\partial_{-} \tilde{b}_{R 1}^{t}\right)-\left(\partial_{-} \tilde{b}_{R 1}^{t}\right) \tilde{a}_{R 1}-\left(\partial_{-} \tilde{c}_{R 1}\right) \tilde{b}_{R 1}^{t}\right]\right\} \\
& =\varepsilon \partial_{-} b_{R 1}^{t}+\varepsilon^{2}\left\{\partial_{-} b_{R 2}^{t}-\left(\frac{3 b_{R 1}^{t}}{2}+B_{R 1}^{t}\right)\left(\partial_{-} a_{R 1}\right)-\left(\frac{3 c_{R 1}}{2}+C_{R 1}\right)\left(\partial_{-} b_{R 1}^{t}\right)\right. \\
& \left.+\left(\partial_{-} b_{R 1}^{t}\right)\left(\frac{3 a_{R 1}}{2}+A_{R 1}\right)+\left(\partial_{-} c_{R 1}\right)\left(\frac{3 b_{R 1}^{t}}{2}+B_{R 1}^{t}\right)\right\}
\end{aligned}
$$

It is apparent that these currents are conserved.

\section{Cartan Decomposition of Symmetric Spaces}

We saw in the above example that symmetric spaces can be decomposed into two pieces, one piece remains invariant under transformation $\mathrm{T}$ though the other piece is transformed in such a way that it behaves like a new symmetric space. Let $\pi$ be the projection $G \longrightarrow M$, sending each $g \in G$ to submersion $M$. We see that $M$ is symmetric space after invariant parts of $G$ are eliminated.

Let $H$ be a closed subgroup of a connected Lie group $\mathrm{G}$, and $\sigma$ be an involutive automorphism of $\mathrm{G}$ such that $F_{0} \subset H \subset F=F i x(\sigma)$. Symmetric space $M$ is the coset space $M=G / H$. If $\mathbf{g}$ is the Lie algebra of $G, \mathbf{h}$ is 
the Lie algebra of $H$, and $\mathbf{m}$ is the Lie subspace 5 for $M$, then $\mathbf{g}=\mathbf{m} \oplus \mathbf{h}$, where $\mathbf{h}$ is closed under brackets while $\mathbf{m}$ is $A d(H)$-invariant subspace of $\mathbf{g}$, i.e, $A d_{h}(\mathbf{m}) \subset \mathbf{m}$ for all $h \in H$. If $X \in \mathbf{g}$, then $X=X_{h}+X_{m}$, where $X_{h} \in \mathbf{h}$, and $X_{m} \in \mathbf{m}$. The involutive automorphism $d \sigma$ is such that $d \sigma\left(X_{h}\right)=X_{h}$ and $d \sigma\left(X_{m}\right)=-X_{m}$. Bracket relations for the symmetric space are defined by

$$
[\mathbf{h}, \mathbf{h}] \subset \mathbf{h}, \quad[\mathbf{h}, \mathbf{m}] \subset \mathbf{m}, \quad[\mathbf{m}, \mathbf{m}] \subset \mathbf{h}
$$

The currents $J_{+}^{(L)}=g^{-1} \partial_{+} g$ and $J_{-}^{(R)}=\left(\partial_{-} g\right) g^{-1}$ on $\mathbf{g}$ can be split into the currents $J_{m}^{(L)}=g^{-1} D_{+} g$ and $J_{m}^{(R)}=\left(D_{-} g\right) g^{-1}$ on $\mathbf{m}$ and $J_{h}^{(L)}=A_{+}$and $J_{h}^{(R)}=g A_{-} g^{-1}$ on $\mathbf{h}$, where $D_{ \pm}$is the covariant derivative acting on $\mathbf{m}$, and $A_{ \pm}$is the gauge field defined on $\mathbf{h}$.

If one defines indices $i, j, k, \ldots$ for the space elements of $\mathbf{g}$, indices $a, b, c, \ldots$ for the space elements of $\mathbf{h}$, and indices $\alpha, \beta, \gamma, \ldots$ for the space elements of $\mathbf{m}$, then (39) allows only structure constants $f_{b c}^{a}, f_{a \beta}^{\alpha}, f_{\beta a}^{\alpha}$, and $f_{\alpha \beta}^{a}$. The other structure constants vanish. This leads to the following equations of motion,

$$
\begin{array}{lll}
k_{+}=g^{-1} D_{+} g & \Longrightarrow & D_{-} k_{+}=0 \\
k_{-}=g^{-1} D_{-} g & \Longrightarrow & D_{+} k_{-}=\left[k_{-}, A_{+}\right]+\left[A_{-}, k_{+}\right] \\
A_{+}=g^{-1} D_{+}^{\prime} g & \Longrightarrow & D_{-}^{\prime} A_{+}=0 \\
A_{-}=g^{-1} D_{-}^{\prime} g & \Longrightarrow & D_{+}^{\prime} A_{-}=\left[A_{-}, A_{+}\right]+\left[k_{-}, k_{+}\right]
\end{array}
$$

where $k_{ \pm}\left(A_{ \pm}\right)$belongs to $\mathbf{m}(\mathbf{h})$, and $D\left(D^{\prime}\right)$ is the covariant derivative acting on $\mathbf{m}(\mathbf{h})$.

It is natural to write down the Pseudoduality equations (41) and (5) in the most general split form on two spaces $\mathbf{m}$ and $\mathbf{h}$ as follows

$$
\begin{array}{cc}
\tilde{k}_{+}^{\alpha}=T_{\beta}^{\alpha} k_{+}^{\beta}+T_{a}^{\alpha} A_{+}^{a} & \tilde{A}_{+}^{a}=T_{b}^{a} A_{+}^{b}+T_{\alpha}^{a} k_{+}^{\alpha} \\
\tilde{k}_{-}^{\alpha}=-T_{\beta}^{\alpha} k_{-}^{\beta}-T_{a}^{\alpha} A_{-}^{a} & \tilde{A}_{-}^{a}=-T_{b}^{a} A_{-}^{b}-T_{\alpha}^{a} k_{-}^{\alpha}
\end{array}
$$

where

$$
\begin{gathered}
g^{-1} \partial_{+} g=\left(\begin{array}{c}
k_{+} \\
A_{+}
\end{array}\right) \quad \begin{array}{l}
\text { on } \mathbf{m} \text {-space } \\
\text { on } \mathbf{h}-\text { space }
\end{array} \\
g^{-1} \partial_{-} g=\left(\begin{array}{c}
k_{-} \\
A_{-}
\end{array}\right) \quad \begin{array}{l}
\text { on } \mathbf{m}-\text { space } \\
\text { on } \mathbf{h} \text {-space }
\end{array}
\end{gathered}
$$

\footnotetext{
${ }^{5} \mathbf{m}$ is called as the Lie subspace for $M$, not Lie Algebra [7].
} 
and

$$
T=\left(\begin{array}{cc}
T_{\beta}^{\alpha} & T_{a}^{\alpha} \\
T_{\beta}^{a} & T_{b}^{a}
\end{array}\right) \quad \begin{array}{cc}
\text { on } & \mathbf{m}-\text { space } \\
\text { on } & \mathbf{h}-\text { space }
\end{array}
$$

Apparently $T_{a}^{\alpha}$ and $T_{\beta}^{a}$ represent the mixing components of the isometry preserving map $T$. Before considering this most general pseudoduality relations which lead to mixed expressions it is worth to analyze pseudoduality equations between pure symmetric spaces and their counter $H$-spaces without mixing parts.

\subsection{Non-Mixing Pseudoduality}

We set the mixing components $T_{a}^{\alpha}$ and $T_{\beta}^{a}$ in equation (44) equal to zero, and consider the pseudoduality equations on $\mathbf{m}$ and $\mathbf{h}$-spaces as follows

$$
\begin{gathered}
\tilde{k}_{ \pm}^{\alpha}= \pm T_{\beta}^{\alpha} k_{ \pm}^{\beta} \\
\tilde{A}_{ \pm}^{a}= \pm T_{b}^{a} A_{ \pm}^{b}
\end{gathered}
$$

When we take $D_{-}$of (48), and $D_{-}^{\prime}$ of (49) ('+' equations only) followed by the equations of motion (40) and (42) we obtain the result that both $T_{\beta}^{\alpha}$ and $T_{b}^{a}$ depend only on $\sigma^{+}$. Now let us take $D_{+}$of '-' equation in (48), and use (41) to get

$$
\left[\tilde{k}_{-}, \tilde{A}_{+}\right]^{\alpha}+\left[\tilde{A}_{-}, \tilde{k}_{+}\right]^{\alpha}=-\left(D_{+} T_{\beta}^{\alpha}\right) k_{-}^{\beta}-T_{\beta}^{\alpha}\left[k_{-}, A_{+}\right]^{\beta}-T_{\beta}^{\alpha}\left[A_{-}, k_{+}\right]^{\beta}
$$

Since $k_{-}$and $A_{-}$can be treated independently, this equation can be split into the following equations

$$
\begin{aligned}
\tilde{f}_{\beta a}^{\alpha} \tilde{k}_{+}^{\beta} T_{c}^{a} & =T_{\beta}^{\alpha} f_{\lambda c}^{\beta} k_{+}^{\lambda} \\
\tilde{f}_{a \beta}^{\alpha} \tilde{A}_{+}^{a} T_{\lambda}^{\beta} & =-D_{+} T_{\lambda}^{\alpha}+T_{\beta}^{\alpha} f_{a \lambda}^{\beta} A_{+}^{a}
\end{aligned}
$$

First equation (51) gives us a relation between structure constants, $\tilde{f}_{\beta a}^{\alpha} T_{\lambda}^{\beta} T_{c}^{a}=$ $T_{\beta}^{\alpha} f_{\lambda c}^{\beta}$, which leads second equation to yield $D_{+} T_{\lambda}^{\alpha}=0$. Therefore we conclude that $T_{\beta}^{\alpha}$ has to be a constant, and we choose it to be identity. Similarly we take $D_{+}^{\prime}$ of '-' equation in (49), and use (43) to get

$$
\left[\tilde{A}_{-}, \tilde{A}_{+}\right]^{a}+\left[\tilde{k}_{-}, \tilde{k}_{+}\right]^{a}=-\left(D_{+}^{\prime} T_{b}^{a}\right) A_{-}^{b}-T_{b}^{a}\left[A_{-}, A_{+}\right]^{b}-T_{b}^{a}\left[k_{-}, k_{+}\right]^{b}
$$


This equation yields the following results

$$
\begin{aligned}
\tilde{f}_{\alpha \beta}^{a} \tilde{k}_{+}^{\alpha} T_{\lambda}^{\beta} & =T_{b}^{a} f_{\beta \lambda}^{b} k_{+}^{\beta} \\
\tilde{f}_{b c}^{a} \tilde{A}_{+}^{b} T_{d}^{c} & =-D_{+}^{\prime} T_{d}^{a}+T_{b}^{a} f_{c d}^{b} A_{+}^{c}
\end{aligned}
$$

First equation (54) verifies the result above up to the permutation of indices, $\tilde{f}_{\alpha \beta}^{a} T_{\nu}^{\alpha} T_{\lambda}^{\beta}=T_{b}^{a} f_{\nu \lambda}^{b}$. Second equation (551) produces the following solution

$$
T_{b}^{a}=T_{b}^{a}(0)+\left(f_{c b}^{a}-\tilde{f}_{c b}^{a}\right) \int_{0}^{\sigma^{+}} A_{+}^{c} D^{\prime} \sigma^{\prime+}+\text { H.O. }
$$

where we choose $T_{b}^{a}(0)$ to be identity. It is easy to see that these equations yield the following bracket relations

$$
\begin{aligned}
{\left[\tilde{k}_{+}, \tilde{A}_{-}\right]^{\alpha} } & =-T_{\beta}^{\alpha}\left[k_{+}, A_{-}\right]^{\beta} \\
{\left[\tilde{k}_{-}, \tilde{A}_{+}\right]^{\alpha} } & =-T_{\beta}^{\alpha}\left[k_{-}, A_{+}\right]^{\beta} \\
{\left[\tilde{k}_{+}, \tilde{k}_{-}\right]^{a} } & =-T_{b}^{a}\left[k_{+}, k_{-}\right]^{b} \\
{\left[\tilde{A}_{+}, \tilde{A}_{-}\right]^{a} } & =-T_{b}^{a}\left[A_{+}, A_{-}\right]^{b}+\left(D_{+}^{\prime} T_{b}^{a}\right) A_{-}^{b}
\end{aligned}
$$

that verifies the equations of motion on pseudodual space as pointed out above, $D_{+} \tilde{k}_{-}^{\alpha}=-T_{\beta}^{\alpha} D_{+} k_{-}^{\beta}$ and $D_{+} \tilde{A}_{-}^{a}=-T_{b}^{a} D_{+}^{\prime} A_{-}^{b}-\left(D_{+}^{\prime} T_{b}^{a}\right) A_{-}^{b}$. We notice that if $H$ and $\tilde{H}$ are the same for both manifolds, i.e., $f_{b c}^{a}=\tilde{f}_{b c}^{a}$, then $T_{b}^{a}$ reduces to identity, and we recover the flat space pseudoduality relations on two manifolds. One can easily construct nonlocal field expressions using above solutions, which are

$$
\begin{aligned}
& \tilde{k}_{ \pm}= \pm k_{ \pm} \\
& \tilde{A}_{ \pm}= \pm A_{ \pm} \pm \int_{0}^{\sigma^{+}}\left(\left[A_{+}\left(\sigma^{\prime}\right), A_{ \pm}\left(\sigma^{+}\right)\right]_{H}-\left[A_{+}\left(\sigma^{\prime}\right), A_{ \pm}\left(\sigma^{+}\right)\right]_{\tilde{H}}\right) D^{\prime} \sigma^{\prime+}+\text { H.O. }
\end{aligned}
$$

One may readily construct nonlocal expressions of the conserved pseudodual currents by means of these fields and following the method in section 2 (??).

\subsection{Mixing Pseudoduality}

We now consider mixing of $\mathbf{m}$ and $\mathbf{h}$-spaces in pseudodual expressions. Pseudoduality equations can be written as in (44). We take $\partial_{-}$of first equation on $\mathbf{m}$-space (44), and obtain

$$
\left(\partial_{-} T_{\beta}^{\alpha}\right) k_{+}^{\beta}+\left(\partial_{-} T_{a}^{\alpha}\right) A_{+}^{a}=0
$$


since $\mathbf{m}$ and $\mathbf{h}$-spaces are independent, we get $\partial_{-} T_{\beta}^{\alpha}=\partial_{-} T_{a}^{\alpha}=0$, so $T_{\beta}^{\alpha}$ and $T_{a}^{\alpha}$ don't depend on $\sigma^{-}$. Now we take $\partial_{+}$of second equation on $\mathbf{m}$-space (44) and see that

$$
\begin{aligned}
{\left[\tilde{k}_{-}, \tilde{A}_{+}\right]^{\alpha}+\left[\tilde{A}_{-}, \tilde{k}_{+}\right]^{\alpha}=} & -\left(\partial_{+} T_{\beta}^{\alpha}\right) k_{-}^{\beta}-T_{\beta}^{\alpha}\left[k_{-}, A_{+}\right]^{\beta}-T_{\beta}^{\alpha}\left[A_{-}, k_{+}\right]^{\beta} \\
& -\left(\partial_{+} T_{a}^{\alpha}\right) A_{-}^{a}-T_{a}^{\alpha}\left[A_{-}, A_{+}\right]^{a}-T_{a}^{\alpha}\left[k_{-}, k_{+}\right]^{a}
\end{aligned}
$$

We substitute the expressions for $\tilde{k}_{-}$and $\tilde{A}_{-}$into this equation, and compare the coefficients of $k_{-}$and $A_{-}$to get the following expressions

$$
\begin{aligned}
\partial_{+} T_{\lambda}^{\alpha} & =\left[f_{b \lambda}^{\beta} T_{\beta}^{\alpha}-\tilde{f}_{a \beta}^{\alpha}\left(T_{b}^{a} T_{\lambda}^{\beta}-T_{b}^{\beta} T_{\lambda}^{a}\right)\right] A_{+}^{b}+\left[f_{\beta \lambda}^{a} T_{a}^{\alpha}-\tilde{f}_{a \nu}^{\alpha}\left(T_{\beta}^{a} T_{\lambda}^{\nu}-T_{\beta}^{\nu} T_{\lambda}^{a}\right)\right] k_{+}^{\beta} \\
\partial_{+} T_{b}^{\alpha} & =\left[f_{\beta b}^{\nu} T_{\nu}^{\alpha}-\tilde{f}_{a \nu}^{\alpha}\left(T_{\beta}^{a} T_{b}^{\nu}-T_{\beta}^{\nu} T_{b}^{a}\right)\right] k_{+}^{\beta}+\left[f_{c b}^{a} T_{a}^{\alpha}-\tilde{f}_{\beta a}^{\alpha}\left(T_{c}^{\beta} T_{b}^{a}-T_{c}^{a} T_{b}^{\beta}\right)\right] A_{+}^{c}
\end{aligned}
$$

Since we only need to find currents up to the second order terms, it suffices to find mapping tensors using only initial values

$$
\begin{aligned}
T_{\lambda}^{\alpha}\left(\sigma^{+}\right)= & T_{\lambda}^{\alpha}(0)+\left(f_{b \lambda}^{\alpha}-\tilde{f}_{b \lambda}^{\alpha}+\tilde{f}_{a \beta}^{\alpha} T_{b}^{\beta}(0) T_{\lambda}^{a}(0)\right) \int_{0}^{\sigma^{+}} A_{+}^{b} D^{\prime} \sigma^{\prime} \\
& +\left(f_{\beta \lambda}^{a} T_{a}^{\alpha}(0)-\tilde{f}_{a \lambda}^{\alpha} T_{\beta}^{a}(0)+\tilde{f}_{a \beta}^{\alpha} T_{\lambda}^{a}(0)\right) \int_{0}^{\sigma^{+}} k_{+}^{\beta} D \sigma^{\prime+}+H . O . \\
T_{b}^{\alpha}\left(\sigma^{+}\right)= & T_{b}^{\alpha}(0)+\left(f_{\beta b}^{\alpha}+\tilde{f}_{b \beta}^{\alpha}-\tilde{f}_{a \nu}^{\alpha} T_{\beta}^{a}(0) T_{b}^{\nu}(0)\right) \int_{0}^{\sigma^{+}} k_{+}^{\beta} D \sigma^{\prime} \\
& +\left(f_{c b}^{a} T_{a}^{\alpha}(0)-\tilde{f}_{\beta b}^{\alpha} T_{c}^{\beta}(0)+\tilde{f}_{\beta c}^{\alpha} T_{b}^{\beta}(0)\right) \int_{0}^{\sigma^{+}} A_{+}^{c} D^{\prime} \sigma^{\prime}+\text { H.O. }
\end{aligned}
$$

where all initial values are chosen to be identity. Therefore pseudodual nonlocal currents on $\tilde{\mathbf{m}}$ can be written as

$$
\begin{aligned}
\tilde{k}_{+}^{\alpha}= & k_{+}^{\alpha}+T_{b}^{\alpha}(0) A_{+}^{b}+\left(f_{\beta \lambda}^{a} T_{a}^{\alpha}(0)-\tilde{f}_{a \lambda}^{\alpha} T_{\beta}^{a}(0)+\tilde{f}_{a \beta}^{\alpha} T_{\lambda}^{a}(0)\right) k_{+}^{\lambda} \int_{0}^{\sigma^{+}} k_{+}^{\beta} D \sigma^{\prime} \\
& +\left(f_{b \beta}^{\alpha}-\tilde{f}_{b \beta}^{\alpha}+\tilde{f}_{a \nu}^{\alpha} T_{b}^{\nu}(0) T_{\beta}^{a}(0)\right) \int_{0}^{\sigma^{+}}\left(A_{+}^{b}\left(\sigma^{\prime}\right) k_{+}^{\beta}\left(\sigma^{+}\right)-k_{+}^{\beta}\left(\sigma^{\prime+}\right) A_{+}^{b}\left(\sigma^{+}\right)\right) d \sigma^{\prime+} \\
& +\left(f_{c b}^{a} T_{a}^{\alpha}(0)-\tilde{f}_{\beta b}^{\alpha} T_{c}^{\beta}(0)+\tilde{f}_{\beta c}^{\alpha} T_{b}^{\beta}(0)\right) A_{+}^{b} \int_{0}^{\sigma^{+}} A_{+}^{c} D^{\prime} \sigma^{\prime}+\text { H.O. }
\end{aligned}
$$




$$
\begin{aligned}
\tilde{k}_{-}^{\alpha}= & -k_{-}^{\alpha}-T_{b}^{\alpha}(0) A_{-}^{b}-\left(f_{\beta \lambda}^{a} T_{a}^{\alpha}-\tilde{f}_{a \lambda}^{\alpha} T_{\beta}^{a}(0)+\tilde{f}_{a \beta}^{\alpha} T_{\lambda}^{a}(0)\right) k_{-}^{\lambda} \int_{0}^{\sigma^{+}} k_{+}^{\beta} D \sigma^{\prime} \\
& +\left(f_{\beta b}^{\alpha}+\tilde{f}_{b \beta}^{\alpha}-\tilde{f}_{a \nu}^{\alpha} T_{b}^{\nu}(0) T_{\beta}^{a}(0)\right) \int_{0}^{\sigma^{+}}\left(A_{+}^{b}\left(\sigma^{\prime}\right) k_{-}^{\beta}\left(\sigma^{+}\right)-k_{+}^{\beta}\left(\sigma^{\prime+}\right) A_{-}^{b}\left(\sigma^{+}\right)\right) d \sigma^{\prime+} \\
& -\left(f_{c b}^{a} T_{a}^{\alpha}(0)-\tilde{f}_{\beta b}^{\alpha} T_{c}^{\beta}(0)+\tilde{f}_{\beta c}^{\alpha} T_{b}^{\beta}(0)\right) A_{-}^{b} \int_{0}^{\sigma^{+}} A_{+}^{c} D^{\prime} \sigma^{\prime}+H . O .
\end{aligned}
$$

Conservation laws of these currents up to the second order terms are obvious. Now we consider pseudoduality equations on h-space (44). We take $\partial_{-}$of first equation, and we obtain

$$
\left(\partial_{-} T_{b}^{a}\right) A_{+}^{b}+\left(\partial_{-} T_{\alpha}^{a}\right) k_{+}^{\alpha}=0
$$

Hence we get $\partial_{-} T_{b}^{a}=\partial_{-} T_{\alpha}^{a}=0$, which implies that $T_{b}^{a}$ and $T_{\alpha}^{a}$ don't depend on $\sigma^{-}$. Taking $\partial_{+}$of second equation we get the following equation

$$
\begin{aligned}
{\left[\tilde{A}_{-}, \tilde{A}_{+}\right]^{a}+\left[\tilde{k}_{-}, \tilde{k}_{+}\right]^{a}=} & -\left(\partial_{+} T_{b}^{a}\right) A_{-}^{b}-T_{b}^{a}\left[A_{-}, A_{+}\right]^{b}-T_{b}^{a}\left[k_{-}, k_{+}\right]^{b} \\
& -\left(\partial_{+} T_{\alpha}^{a}\right) k_{-}^{\alpha}-T_{\alpha}^{a}\left[k_{-}, A_{+}\right]^{\alpha}-T_{\alpha}^{a}\left[A_{-}, k_{+}\right]^{\alpha}
\end{aligned}
$$

We replace $\tilde{A}_{-}$and $\tilde{k}_{-}$in this equation to obtain the following results

$$
\begin{aligned}
& \partial_{+} T_{d}^{a}=\left(T_{b}^{a} f_{e d}^{b}-\tilde{f}_{b c}^{a} T_{e}^{b} T_{d}^{c}-\tilde{f}_{\alpha \beta}^{a} T_{e}^{\alpha} T_{d}^{\beta}\right) A_{+}^{e}+\left(T_{\alpha}^{a} f_{\lambda d}^{\alpha}-\tilde{f}_{b c}^{a} T_{\lambda}^{b} T_{d}^{c}-\tilde{f}_{\alpha \beta}^{a} T_{\lambda}^{\alpha} T_{d}^{\beta}\right) k_{+}^{\lambda} \\
& \partial_{+} T_{\nu}^{a}=\left(T_{b}^{a} f_{\lambda \nu}^{b}-\tilde{f}_{b c}^{a} T_{\lambda}^{b} T_{\nu}^{c}-\tilde{f}_{\alpha \beta}^{a} T_{\lambda}^{\alpha} T_{\nu}^{\beta}\right) k_{+}^{\lambda}+\left(T_{\alpha}^{a} f_{d \nu}^{\alpha}-\tilde{f}_{b c}^{a} T_{d}^{b} T_{\nu}^{c}-\tilde{f}_{\alpha \beta}^{a} T_{d}^{\alpha} T_{\nu}^{\beta}\right) A_{+}^{d}
\end{aligned}
$$

We again want to find solutions up to the second order terms, so we only use initial values to get

$$
\begin{aligned}
T_{d}^{a}\left(\sigma^{+}\right)= & T_{d}^{a}(0)+\left(f_{e d}^{a}-\tilde{f}_{e d}^{a}-\tilde{f}_{\alpha \beta}^{a} T_{e}^{\alpha}(0) T_{d}^{\beta}(0)\right) \int_{0}^{\sigma^{+}} A_{+}^{e} D^{\prime} \sigma^{\prime} \\
& +\left(T_{\alpha}^{a}(0) f_{\lambda d}^{\alpha}-\tilde{f}_{b d}^{a} T_{\lambda}^{b}(0)-\tilde{f}_{\lambda \beta}^{a} T_{d}^{\beta}(0)\right) \int_{0}^{\sigma^{+}} k_{+}^{\lambda} D \sigma^{\prime+}+H . O . \\
T_{\nu}^{a}\left(\sigma^{+}\right)= & T_{\nu}^{a}(0)+\left(f_{\lambda \nu}^{a}-\tilde{f}_{\lambda \nu}^{a}-\tilde{f}_{b c}^{a} T_{\lambda}^{b}(0) T_{\nu}^{c}(0)\right) \int_{0}^{\sigma^{+}} k_{+}^{\lambda} D \sigma^{\prime} \\
& +\left(T_{\alpha}^{a}(0) f_{d \nu}^{\alpha}-\tilde{f}_{d c}^{a} T_{\nu}^{c}(0)-\tilde{f}_{\alpha \nu}^{a} T_{d}^{\alpha}(0)\right) \int_{0}^{\sigma^{+}} A_{+}^{d} D^{\prime} \sigma^{\prime}+\text { H.O. }
\end{aligned}
$$


Thus pseudodual fields up to the second order terms on $H$ space will be

$$
\begin{aligned}
\tilde{A}_{+}^{a} & =A_{+}^{a}+T_{\lambda}^{a}(0) k_{+}^{\lambda}+\left(f_{e d}^{a}-\tilde{f}_{e d}^{a}-\tilde{f}_{\alpha \beta}^{a} T_{e}^{\alpha}(0) T_{d}^{\beta}(0)\right) A_{+}^{d} \int_{0}^{\sigma^{+}} A_{+}^{e} D^{\prime} \sigma^{\prime}+ \\
& +\left(T_{\alpha}^{a}(0) f_{\lambda d}^{\alpha}-\tilde{f}_{b d}^{a} T_{\lambda}^{b}(0)-\tilde{f}_{\lambda \beta}^{a} T_{d}^{\beta}(0)\right) \int_{0}^{\sigma^{+}}\left(k_{+}^{\lambda}\left(\sigma^{\prime}\right) A_{+}^{d}\left(\sigma^{+}\right)-A_{+}^{d}\left(\sigma^{\prime+}\right) k_{+}^{\lambda}\left(\sigma^{+}\right)\right) d \sigma^{\prime+} \\
& +\left(f_{\lambda \nu}^{a}-\tilde{f}_{\lambda \nu}^{a}-\tilde{f}_{b c}^{a} T_{\lambda}^{b}(0) T_{\nu}^{c}(0)\right) k_{+}^{\nu} \int_{0}^{\sigma^{+}} k_{+}^{\lambda} D \sigma^{\prime+}+H . O . \\
\tilde{A}_{-}^{a} & =-A_{-}^{a}-T_{\lambda}^{a}(0) k_{-}^{\lambda}-\left(f_{e d}^{a}-\tilde{f}_{e d}^{a}-\tilde{f}_{\alpha \beta}^{a} T_{e}^{\alpha}(0) T_{d}^{\beta}(0)\right) A_{-}^{d} \int_{0}^{\sigma^{+}} A_{+}^{e} D^{\prime} \sigma^{\prime+} \\
& -\left(T_{\alpha}^{a}(0) f_{\lambda d}^{\alpha}-\tilde{f}_{b d}^{a} T_{\lambda}^{b}(0)-\tilde{f}_{\lambda \beta}^{a} T_{d}^{\beta}(0)\right) \int_{0}^{\sigma^{+}}\left(k_{+}^{\lambda}\left(\sigma^{\prime}\right) A_{-}^{d}\left(\sigma^{+}\right)-A_{+}^{d}\left(\sigma^{\prime}\right) k_{-}^{\lambda}\left(\sigma^{+}\right)\right) d \sigma^{\prime}+ \\
& -\left(f_{\lambda \nu}^{a}-\tilde{f}_{\lambda \nu}^{a}-\tilde{f}_{b c}^{a} T_{\lambda}^{b}(0) T_{\nu}^{c}(0)\right) k_{-}^{\nu} \int_{0}^{\sigma^{+}} k_{+}^{\lambda} D \sigma^{+}+H . O .
\end{aligned}
$$

It is obvious that conservation laws (42) and (43) up to the second order terms are satisfied

$$
\begin{aligned}
\tilde{D}_{-}^{\prime} \tilde{A}_{+}^{a}= & 0 \\
\tilde{D}_{+}^{\prime} \tilde{A}_{-}^{a}= & -\left[A_{-}, A_{+}\right]_{\tilde{G}}^{a}-\left[k_{-}, k_{+}\right]_{\tilde{G}}^{a}-\left[T(0) A_{-}, T(0) A_{+}\right]_{\tilde{G}}^{a}-\left[A_{-}, T(0) k_{+}\right]_{\tilde{G}}^{a} \\
& -\left[T(0) k_{-}, A_{+}\right]_{\tilde{G}}^{a}-\left[T(0) A_{-}, k_{+}\right]_{\tilde{G}}^{a}-\left[k_{-}, T(0) A_{+}\right]_{\tilde{G}}^{a} \\
& -\left[T(0) k_{-}, T(0) k_{+}\right]_{\tilde{G}}^{a}+H . O .
\end{aligned}
$$

\subsection{Dual Symmetric Spaces and Further Constraints}

It is well-known [3, 7] that two normal symmetric spaces are dual symmetric spaces if there exist

1. a Lie algebra isomorphism $S: \mathbf{h} \longrightarrow \tilde{\mathbf{h}}$ such that $\tilde{Q}(S V, S W)=$ $-Q(V, W)$ for all $V, W \in \mathbf{h}$, and $Q$ is inner product.

2. a linear isometry $T: \mathbf{m} \longrightarrow \tilde{\mathbf{m}}$ such that $[T X, T Y]=-S[X, Y]$ for all $X, Y \in \mathbf{m}$.

Item (1) tells us that brackets in $\mathbf{h}$ and $\tilde{\mathbf{h}}$ are the same while item (2) tells us that inner products in $\mathbf{m}$ and $\tilde{\mathbf{m}}$ are the same. Item (1) yields the result 
$f_{c b}^{a}=\tilde{f}_{c b}^{a}$ for non-mixing pseudoduality, which leads $T_{b}^{a}$ to be a constant. Hence pseudoduality transformations will simply be

$$
\begin{gathered}
\tilde{k}_{ \pm}^{\alpha}= \pm k_{ \pm}^{\alpha} \\
\tilde{A}_{ \pm}^{a}= \pm A_{ \pm}^{a}
\end{gathered}
$$

with the bracket relations (57) $-(\underline{601})$ given by

$$
\begin{aligned}
{\left[\tilde{k}_{+}, \tilde{A}_{-}\right]^{\alpha} } & =-\left[k_{+}, A_{-}\right]^{\alpha} \\
{\left[\tilde{k}_{-}, \tilde{A}_{+}\right]^{\alpha} } & =-\left[k_{-}, A_{+}\right]^{\alpha} \\
{\left[\tilde{k}_{+}, \tilde{k}_{-}\right]^{a} } & =-\left[k_{+}, k_{-}\right]^{a} \\
{\left[\tilde{A}_{+}, \tilde{A}_{-}\right]^{a} } & =-\left[A_{+}, A_{-}\right]^{a}
\end{aligned}
$$

On the other hand one can write the following bracket relations between pseudodual target spaces for the mixing pseudoduality case

$$
\begin{aligned}
{\left[\tilde{k}_{-}, \tilde{A}_{+}\right]^{\alpha}+\left[\tilde{A}_{-}, \tilde{k}_{+}\right]^{\alpha}=} & -T_{\beta}^{\alpha}\left[k_{-}, A_{+}\right]^{\beta}-T_{\beta}^{\alpha}\left[A_{-}, k_{+}\right]^{\beta} \\
& -T_{a}^{\alpha}\left[A_{-}, A_{+}\right]^{a}-T_{a}^{\alpha}\left[k_{-}, k_{+}\right]^{a} \\
{\left[\tilde{A}_{-}, \tilde{A}_{+}\right]^{a}+\left[\tilde{k}_{-}, \tilde{k}_{+}\right]^{a}=} & -T_{b}^{a}\left[A_{-}, A_{+}\right]^{b}-T_{b}^{a}\left[k_{-}, k_{+}\right]^{b} \\
& -T_{\alpha}^{a}\left[k_{-}, A_{+}\right]^{\alpha}-T_{\alpha}^{a}\left[A_{-}, k_{+}\right]^{\alpha}
\end{aligned}
$$

which in turn leads to relations of connection two-forms between symmetric and corresponding $\mathrm{H}$-spaces, which is consistent with the result found in section 5 (??). These equations produce that all components of the pseudoduality map $T$ must be constant, and we choose them to be identity. Hence pseudoduality equations will simply be

$$
\begin{aligned}
\tilde{k}_{ \pm}^{\alpha} & = \pm k_{ \pm}^{\alpha} \pm T_{a}^{\alpha}(0) A_{ \pm}^{a} \\
\tilde{A}_{ \pm}^{a} & = \pm A_{ \pm}^{a} \pm T_{\alpha}^{a}(0) k_{ \pm}^{\alpha}
\end{aligned}
$$

\subsection{An Example}

We consider the Lie groups we used in the previous section. We saw that invariant subspace of $S O(n+1)$ is $1 \times S O(n)$. We pick $H$ space as $S O(n)$. Hence our symmetric space is $M=\frac{S O(n+1)}{S O(n)}$. The Lie algebra $\mathbf{g}=s o(n+1)$ can be written as

$$
\operatorname{so}(n+1)=\left(\begin{array}{cc}
a & b \\
-b^{t} & c
\end{array}\right) \quad \begin{aligned}
& a=1 \times 1 \\
& b=1 \times n \\
& c=n \times n
\end{aligned}
$$


which can be split as

$$
\left(\begin{array}{cc}
a & b \\
-b^{t} & c
\end{array}\right)=\left(\begin{array}{cc}
a & 0 \\
0 & c
\end{array}\right)+\left(\begin{array}{cc}
0 & b \\
-b^{t} & 0
\end{array}\right) \quad \mathbf{g}=\mathbf{h} \oplus \mathbf{m}
$$

Let $Y \in \mathbf{g}, X \in \mathbf{h}$, and $Z \in \mathbf{m}$. Then, $D^{\prime} Z=0$ and $D X=0$. Using the expansions (14) and (15), we may write the following expressions

$$
\begin{aligned}
k_{+}^{\alpha} & =D_{+} Z_{L}^{\alpha}-\frac{1}{2}\left[X_{L}, D_{+} Z_{L}\right]^{\alpha}-\frac{1}{2}\left[Z_{L}, D_{+}^{\prime} X_{L}\right]^{\alpha}+H . O . \\
A_{+}^{a} & =D_{+}^{\prime} X_{L}^{a}-\frac{1}{2}\left[X_{L}, D_{+}^{\prime} X_{L}\right]^{a}-\frac{1}{2}\left[Z_{L}, D_{+} Z_{L}\right]^{a}+H . O . \\
k_{-}^{\alpha} & =D_{-} Z_{R}^{\alpha}-\left[X_{L}, D_{-} Z_{R}\right]^{\alpha}-\left[Z_{L}, D_{-}^{\prime} X_{R}\right]^{\alpha}-\frac{1}{2}\left[X_{R}, D_{-} Z_{R}\right]^{\alpha} \\
& -\frac{1}{2}\left[Z_{R}, D_{-}^{\prime} X_{R}\right]^{\alpha}+H . O . \\
A_{-}^{a} & =D_{-}^{\prime} X_{R}^{a}-\left[X_{L}, D_{-}^{\prime} X_{R}\right]^{a}-\left[Z_{L}, D_{-} Z_{R}\right]^{a}-\frac{1}{2}\left[X_{R}, D_{-}^{\prime} X_{R}\right]^{a} \\
& -\frac{1}{2}\left[Z_{R}, D_{-} Z_{R}\right]^{a}+H . O .
\end{aligned}
$$

We describe solutions $X=\sum_{n=1}^{\infty} \varepsilon^{n} x_{n}$ and $Z=\sum_{n=1}^{\infty} \varepsilon^{n} z_{n}$, where $\varepsilon$ is a small parameter. It is clear that equations of motion (40)-(43) for all orders of $\varepsilon$ are satisfied. In the following calculations we are going to use expressions up to the order of $\varepsilon^{2}$ for simplicity.

Now we consider dual symmetric space $\tilde{M}=\frac{S O(n, 1)}{S O(n)}$, where $\tilde{H}=S O(n)$. Lie algebra $\tilde{\mathbf{g}}=s o(n, 1)$ is written as

$$
\operatorname{so}(n, 1)=\left(\begin{array}{cc}
\tilde{a} & \tilde{b} \\
\tilde{b}^{t} & \tilde{c}
\end{array}\right) \quad \begin{aligned}
& \tilde{a}=1 \times 1 \\
& \tilde{b}=1 \times n \\
& \tilde{c}=n \times n
\end{aligned}
$$

which is split as

$$
\left(\begin{array}{cc}
\tilde{a} & \tilde{b} \\
\tilde{b}^{t} & \tilde{c}
\end{array}\right)=\left(\begin{array}{cc}
\tilde{a} & 0 \\
0 & \tilde{c}
\end{array}\right)+\left(\begin{array}{cc}
0 & \tilde{b} \\
\tilde{b}^{t} & 0
\end{array}\right) \quad \tilde{\mathbf{g}}=\tilde{\mathbf{h}} \oplus \tilde{\mathbf{m}}
$$

Let $\tilde{Y}=\tilde{X}+\tilde{Z}$, where $\tilde{Y} \in \tilde{\mathbf{g}}, \tilde{X} \in \tilde{\mathbf{h}}$, and $\tilde{Z} \in \tilde{\mathbf{m}}$. We get the same fields as equations (93)-(96) with tilde. Equations of motion will be the same with tilde. We may now find pseudodual fields using our expressions 
found above. We note that because of the special form of our Lie groups, mixing components of the map $T$ vanishes, and we simply get non-mixing pseudoduality condition.

We insert our expressions into equations (81) and (82) to get infinitely many pseudoduality relations. Up to the order of $\varepsilon^{2}$ terms equation (81) will be

$$
\begin{gathered}
\tilde{D}_{+} \tilde{z}_{L 1}^{\alpha}=D_{+} z_{L 1}^{\alpha} \quad \tilde{D}_{-} \tilde{z}_{R 1}^{\alpha}=-D_{-} z_{R 1}^{\alpha} \\
\tilde{D}_{+} \tilde{z}_{L 2}^{\alpha}-\frac{1}{2}\left[\tilde{x}_{L 1}, \tilde{D}_{+} \tilde{z}_{L 1}\right]^{\alpha}-\frac{1}{2}\left[\tilde{z}_{L 1}, \tilde{D}_{+}^{\prime} \tilde{x}_{L 1}\right]^{\alpha}=D_{+} z_{L 2}^{\alpha}-\frac{1}{2}\left[x_{L 1}, D_{+} z_{L 1}\right]^{\alpha}-\frac{1}{2}\left[z_{L 1}, D_{+}^{\prime} x_{L 1}\right]^{\alpha} \\
\tilde{D}_{-} \tilde{z}_{R 2}^{\alpha}-\left[\tilde{x}_{L 1}, \tilde{D}_{-} \tilde{z}_{R 1}\right]^{\alpha}-\left[\tilde{z}_{L 1}, \tilde{D}_{-}^{\prime} \tilde{x}_{R 1}\right]^{\alpha}-\frac{1}{2}\left[\tilde{x}_{R 1}, \tilde{D}_{-} \tilde{z}_{R 1}\right]^{\alpha}-\frac{1}{2}\left[\tilde{z}_{R 1}, \tilde{D}_{-}^{\prime} \tilde{x}_{R 1}\right]^{\alpha}= \\
-D_{-} z_{R 2}^{\alpha}+\left[x_{L 1}, D_{-} z_{R 1}\right]^{\alpha}+\left[z_{L 1}, D_{-}^{\prime} x_{R 1}\right]^{\alpha}+\frac{1}{2}\left[x_{R 1}, D_{-} z_{R 1}\right]^{\alpha}+\frac{1}{2}\left[z_{R 1}, D_{-}^{\prime} x_{R 1}\right]^{\alpha}
\end{gathered}
$$

and equation (82) will be

$$
\begin{gathered}
\tilde{D}_{+}^{\prime} \tilde{x}_{L 1}^{a}=D_{+}^{\prime} x_{L 1}^{a} \quad \tilde{D}_{-}^{\prime} \tilde{x}_{R 1}^{a}=-D_{-}^{\prime} x_{R 1}^{a} \\
\tilde{D}_{+}^{\prime} \tilde{x}_{L 2}^{a}-\frac{1}{2}\left[\tilde{x}_{L 1}, \tilde{D}_{+}^{\prime} \tilde{x}_{L 1}\right]^{a}-\frac{1}{2}\left[\tilde{z}_{L 1}, \tilde{D}_{+} \tilde{z}_{L 1}\right]^{a}=D_{+}^{\prime} x_{L 2}^{a}-\frac{1}{2}\left[x_{L 1}, D_{+}^{\prime} x_{L 1}\right]^{a}-\frac{1}{2}\left[z_{L 1}, D_{+} z_{L 1}\right]^{a} \\
\tilde{D}_{-}^{\prime} \tilde{x}_{R 2}^{a}-\left[\tilde{x}_{L 1}, \tilde{D}_{-}^{\prime} \tilde{x}_{R 1}\right]^{a}-\left[\tilde{z}_{L 1}, \tilde{D}_{-} \tilde{z}_{R 1}\right]^{a}-\frac{1}{2}\left[\tilde{x}_{R 1}, \tilde{D}_{-}^{\prime} \tilde{x}_{R 1}\right]^{a}-\frac{1}{2}\left[\tilde{z}_{R 1}, \tilde{D}_{-} \tilde{z}_{R 1}\right]^{a}= \\
-D_{-}^{\prime} x_{R 2}^{a}+\left[x_{L 1}, D_{-}^{\prime} x_{R 1}\right]^{a}+\left[z_{L 1}, D_{-} z_{R 1}\right]^{a}+\frac{1}{2}\left[x_{R 1}, D_{-}^{\prime} x_{R 1}\right]^{a}+\frac{1}{2}\left[z_{R 1}, D_{-} z_{R 1}\right]^{a}
\end{gathered}
$$

Since we know

$$
\begin{gathered}
D_{ \pm} z_{n}=\left(\begin{array}{cc}
0 & D_{ \pm} b_{n} \\
-D_{ \pm} b_{n}^{t} & 0
\end{array}\right) \quad D_{ \pm}^{\prime} x_{n}=\left(\begin{array}{cc}
D_{ \pm}^{\prime} a_{n} & 0 \\
0 & D_{ \pm}^{\prime} c_{n}
\end{array}\right) \\
{\left[x_{1}, D_{ \pm}^{\prime} x_{1}\right]=\left(\begin{array}{cc}
{\left[a_{1}, D_{ \pm}^{\prime} a_{1}\right]} & 0 \\
0 & {\left[c_{1}, D_{ \pm}^{\prime} c_{1}\right]}
\end{array}\right)} \\
{\left[z_{1}, D_{ \pm} z_{1}\right]=\left(\begin{array}{cc}
\left(D_{ \pm} b_{1}\right) b_{1}^{t}-b_{1}\left(D_{ \pm} b_{1}^{t}\right) & \left(D_{ \pm} b_{1}^{t}\right) b_{1}-b_{1}^{t}\left(D_{ \pm} b_{1}\right) \\
0 &
\end{array}\right)}
\end{gathered}
$$




$$
\begin{aligned}
& {\left[x_{1}, D_{ \pm} z_{1}\right]=\left(\begin{array}{cc}
0 & a_{1} D_{ \pm} b_{1}-\left(D_{ \pm} b_{1}\right) c_{1} \\
-c_{1}\left(D_{ \pm} b_{1}^{t}\right)+\left(D_{ \pm} b_{1}^{t}\right) a_{1} & 0
\end{array}\right)} \\
& {\left[z_{1}, D_{ \pm}^{\prime} x_{1}\right]=\left(\begin{array}{cc}
0 & b_{1} D_{ \pm}^{\prime} c_{1}-\left(D_{ \pm}^{\prime} a_{1}\right) b_{1} \\
-b_{1}^{t}\left(D_{ \pm}^{\prime} a_{1}\right)+\left(D_{ \pm}^{\prime} c_{1}\right) b_{1}^{t} & 0
\end{array}\right)}
\end{aligned}
$$

One can write similar expressions on the pseudodual space replacing each term with tilded terms. Only exception is that we switch $b_{n}^{t}$ with $-\tilde{b}_{n}^{t}$ so that we get the convenient lie algebra on tilded space. Therefore pseudoduality equations above (99) and (100) will give the following expressions

$$
\begin{gathered}
\tilde{D}_{+} \tilde{b}_{L 1}=D_{+} b_{L 1} \quad \tilde{D}_{+} \tilde{b}_{L 1}^{t}=-D_{+} b_{L 1}^{t} \\
\tilde{D}_{-} \tilde{b}_{R 1}=-D_{-} b_{R 1} \quad \tilde{D}_{-} \tilde{b}_{R 1}^{t}=D_{-} b_{R 1}^{t} \\
\tilde{D}_{+}^{\prime} \tilde{a}_{L 1}=D_{+}^{\prime} a_{L 1} \quad \tilde{D}_{+}^{\prime} \tilde{c}_{L 1}=D_{+}^{\prime} c_{L 1} \\
\tilde{D}_{-}^{\prime} \tilde{a}_{R 1}=-D_{-}^{\prime} a_{R 1} \quad \tilde{D}_{-}^{\prime} \tilde{c}_{R 1}=-D_{-}^{\prime} c_{R 1} \\
\tilde{D}_{+} \tilde{b}_{L 2}=D_{+} b_{L 2}+\frac{1}{2}\left\{\left(\tilde{a}_{L 1}-a_{L 1}\right) D_{+} b_{L 1}-D_{+} b_{L 1}\left(\tilde{c}_{L 1}-c_{L 1}\right)\right\} \\
+\frac{1}{2}\left\{\left(\tilde{b}_{L 1}-b_{L 1}\right) D_{+}^{\prime} c_{L 1}-D_{+}^{\prime} a_{L 1}\left(\tilde{b}_{L 1}-b_{L 1}\right)\right\} \\
\tilde{D}_{+} \tilde{b}_{L 2}^{t}=-D_{+} b_{L 2}^{t}-\frac{1}{2}\left\{\left(\tilde{c}_{L 1}-c_{L 1}\right) D_{+} b_{L 1}^{t}-D_{+} b_{L 1}^{t}\left(\tilde{a}_{L 1}-a_{L 1}\right)\right\} \\
+\frac{1}{2}\left\{\left(\tilde{b}_{L 1}^{t}+b_{L 1}^{t}\right) D_{+}^{\prime} a_{L 1}-D_{+}^{\prime} c_{L 1}\left(\tilde{b}_{L 1}^{t}+b_{L 1}^{t}\right)\right\} \\
\tilde{D}_{+}^{\prime} \tilde{a}_{L 2}=D_{+}^{\prime} a_{L 2}+\frac{1}{2}\left[\left(\tilde{a}_{L 1}-a_{L 1}\right), D_{+}^{\prime} a_{L 1}\right] \\
-\frac{1}{2}\left\{D_{+} b_{L 1}\left(b_{L 1}^{t}+\tilde{b}_{L 1}^{t}\right)-\left(b_{L 1}-\tilde{b}_{L 1}\right) D_{+} b_{L 1}^{t}\right\} \\
\tilde{D}_{+}^{\prime} \tilde{c}_{L 2}=D_{+}^{\prime} c_{L 2}+\frac{1}{2}\left[\left(\tilde{c}_{L 1}-c_{L 1}\right), D_{+}^{\prime} c_{L 1}\right] \\
-\frac{1}{2}\left\{D_{+} b_{L 1}^{t}\left(b_{L 1}-\tilde{b}_{L 1}\right)-\left(b_{L 1}^{t}+\tilde{b}_{L 1}^{t}\right) D_{+} b_{L 1}\right\}
\end{gathered}
$$




$$
\begin{aligned}
\tilde{D}_{-} \tilde{b}_{R 2}=-D_{-} b_{R 2}+ & \left\{\left(a_{L 1}-\tilde{a}_{L 1}\right)+\frac{1}{2}\left(a_{R 1}-\tilde{a}_{R 1}\right)\right\} D_{-} b_{R 1} \\
- & D_{-} b_{R 1}\left\{\left(c_{L 1}-\tilde{c}_{L 1}\right)+\frac{1}{2}\left(c_{R 1}-\tilde{c}_{R 1}\right)\right\} \\
+ & \left\{\left(b_{L 1}-\tilde{b}_{L 1}\right)+\frac{1}{2}\left(b_{R 1}-\tilde{b}_{R 1}\right)\right\} D_{-}^{\prime} c_{R 1} \\
& -D_{-}^{\prime} a_{R 1}\left\{\left(b_{L 1}-\tilde{b}_{L 1}\right)+\frac{1}{2}\left(b_{R 1}-\tilde{b}_{R 1}\right)\right\} \\
\tilde{D}_{-} \tilde{b}_{R 2}^{t}=D_{-} b_{R 2}^{t}- & \left\{\left(c_{L 1}-\tilde{c}_{L 1}\right)+\frac{1}{2}\left(c_{R 1}-\tilde{c}_{R 1}\right)\right\} D_{-} b_{R 1}^{t} \\
+ & D_{-} b_{R 1}^{t}\left\{\left(a_{L 1}-\tilde{a}_{L 1}\right)+\frac{1}{2}\left(a_{R 1}-\tilde{a}_{R 1}\right)\right\} \\
- & \left\{\left(b_{L 1}^{t}+\tilde{b}_{L 1}^{t}\right)+\frac{1}{2}\left(b_{R 1}^{t}+\tilde{b}_{R 1}^{t}\right)\right\} D_{-}^{\prime} a_{R 1} \\
+ & D_{-}^{\prime} c_{R 1}\left\{\left(b_{L 1}^{t}+\tilde{b}_{L 1}^{t}\right)+\frac{1}{2}\left(b_{R 1}^{t}+\tilde{b}_{R 1}^{t}\right)\right\} \\
\tilde{D}_{-}^{\prime} \tilde{c}_{R 2}=-D_{-}^{\prime} c_{R 2}+ & {\left[\left(c_{L 1}-\tilde{c}_{L 1}\right)+\frac{1}{2}\left(c_{R 1}-\tilde{c}_{R 1}\right), D_{-}^{\prime} c_{R 1}\right] } \\
+ & D_{-} b_{R 1}^{t}\left\{\left(b_{L 1}-\tilde{b}_{L 1}\right)+\frac{1}{2}\left(b_{R 1}+\tilde{b}_{R 1}\right)\right\} \\
& -\left\{\left(b_{L 1}^{t}+\tilde{b}_{L 1}^{t}\right)+\frac{1}{2}\left(b_{R 1}^{t}+\tilde{b}_{R 1}^{t}\right)\right\} D_{-} b_{R 1} \\
\tilde{D}_{-}^{\prime} \tilde{a}_{R 2}=-D_{-}^{\prime} a_{R 2}+ & {\left[\left(a_{L 1}-\tilde{a}_{L 1}\right)+\frac{1}{2}\left(a_{R 1}-\tilde{a}_{R 1}\right), D_{-}^{\prime} a_{R 1}\right] } \\
+ & D_{-} b_{R 1}\left\{\left(b_{L 1}^{t}+\tilde{b}_{L 1}^{t}\right)+\frac{1}{2}\left(b_{R 1}^{t}+\tilde{b}_{R 1}^{t}\right)\right\} \\
- & \left\{\left(b_{L 1}-\tilde{b}_{L 1}\right)+\frac{1}{2}\left(b_{R 1}-\tilde{b}_{R 1}\right)\right\} D_{-} b_{R 1}^{t} \\
& \\
&
\end{aligned}
$$

where tilded terms on the right hand sides can be replaced by solving corresponding equations. One can obtain the conserved nonlocal currents using these terms. 


\section{Curvatures}

\subsection{Case I: Curvatures on $\mathrm{g}$ and $\tilde{\mathrm{g}}$}

Let us find the curvatures related to symmetric spaces, and see the relations between dual symmetric parts. We first consider the case where $\mathrm{H}=\mathrm{id}$. We may choose orthonormal frame $\{J\}$ on the pullback bundle $g^{*}(T G)$, where $J$ stands for both $J^{(R)}$ and $J^{(L)}$. These currents satisfy the Maurer-Cartan equation

$$
d J^{i}+\frac{1}{2} f_{j k}^{i} J^{j} \wedge J^{k}=0
$$

where $w^{i}=J^{i}$ and $w_{k}^{i}=\frac{1}{2} f_{j k}^{i} J^{j}$ is the antisymmetric riemannian connection. Curvature can be found using torsion free Cartan structural equations

$$
\begin{aligned}
d w^{i}+w_{j}^{i} \wedge w^{j} & =0 \\
d w_{j}^{i}+w_{k}^{i} \wedge w_{j}^{k} & =\frac{1}{2} R_{j k l}^{i} w^{k} \wedge w^{l}
\end{aligned}
$$

Substituting $w^{i}=J^{i}$ and $w_{j}^{i}=\frac{1}{2} f_{k j}^{i} J^{k}$ into first equation gives us the MaurerCartan equation (101). Curvature tensor associated with $\mathrm{g}$ can be found using second equation (103),

$$
R_{j m n}^{i}=-\frac{1}{2}\left(f_{k m}^{i} f_{n j}^{k}+f_{k j}^{i} f_{m n}^{k}\right)=\frac{1}{2} f_{k n}^{i} f_{j m}^{k}
$$

where we used jacobi identity in the last equation, $f_{k[m}^{i} f_{n j]}^{k}=0$. We may find similar relations for pseudodual space with tilde (just put ${ }^{\sim}$ on each term). To relate curvature tensor on pseudodual space with regular space, we use nonlocal expressions (26)-(29). Since both currents yield the same result, we just use (26) and (27) for the final expression. We may write $\tilde{J}^{i}$ in nonlocal terms as

$$
\tilde{J}^{i}=\varepsilon d y_{1}^{i}+\varepsilon^{2}\left[d y_{2}^{i}+\frac{1}{2} f_{j k}^{i} y_{1}^{i} \wedge d y_{1}^{k}-\tilde{f}_{j k}^{i} y_{1}^{j} \wedge d y_{1}^{k}\right]+\text { H.O. }
$$

Hence $\tilde{w}^{i}=\tilde{J}^{i}$, and $\tilde{w}_{k}^{i}$ can be written as

$$
\begin{aligned}
\tilde{w}_{k}^{i} & =\frac{1}{2} \tilde{f}_{j k}^{i} \tilde{J}^{j} \\
& =\frac{\varepsilon}{2} \tilde{f}_{j k}^{i} d y_{1}^{j}+\frac{\varepsilon^{2}}{2} \tilde{f}_{j k}^{i}\left[d y_{2}^{j}+\frac{1}{2} f_{m n}^{j} y_{1}^{m} \wedge d y_{1}^{n}-\tilde{f}_{m n}^{j} y_{1}^{m} \wedge d y_{1}^{n}\right]+H . O .
\end{aligned}
$$


We plug $\tilde{w}^{i}$ and $\tilde{w}_{k}^{i}$ into the second Cartan structural equation on pseudodual space in the form

$$
d \tilde{w}_{j}^{i}+\tilde{w}_{k}^{i} \wedge \tilde{w}_{j}^{k}=\frac{1}{2} \tilde{R}_{j k l}^{i} \tilde{w}^{k} \wedge \tilde{w}^{l}
$$

to obtain the curvature expression

$$
\tilde{R}_{j m n}^{i}=\frac{1}{2} \tilde{f}_{k j}^{i} f_{m n}^{k}-\tilde{f}_{k j}^{i} \tilde{f}_{m n}^{k}+\frac{1}{2} \tilde{f}_{m k}^{i} \tilde{f}_{n j}^{k}
$$

Since by definition $\tilde{R}_{j m n}^{i}(\underline{104})$ can also be written as

$$
\tilde{R}_{j m n}^{i}=\frac{1}{2} \tilde{f}_{k n}^{i} \tilde{f}_{j m}^{k}
$$

we get a relation between structure constants on spaces $\mathbf{g}$ and $\tilde{\mathbf{g}}$

$$
\frac{1}{2} \tilde{f}_{k j}^{i} f_{m n}^{k}=\frac{1}{2} \tilde{f}_{k j}^{i} \tilde{f}_{m n}^{k}
$$

where we used the jacobi identity $\tilde{f}_{k[n}^{i} \tilde{f}_{j m]}^{k}=0$. Though we do not set $f_{m n}^{k}$ equal to $\tilde{f}_{m n}^{k}$, we may treat them on equal footing, and use one for another interchangeably in paired terms. Hence $\tilde{R}_{j m n}^{i}(108)$ can be written in nonlocal structure constants as

$$
\tilde{R}_{j m n}^{i}=-\frac{1}{2} f_{k n}^{i} f_{j m}^{k}=-R_{j m n}^{i}
$$

where we used $f_{k[j}^{i} f_{n m]}^{k}=0$ after setting tilde terms with nontilde terms. We note that we obtained pseudodual space curvature as the negative regular space curvature. This shows that spaces are dual symmetric spaces as we expressed above.

\subsection{Case II: Curvatures on Decomposed Spaces}

Let us decompose the current as $J=J^{\alpha} t_{\alpha}+J^{a} t_{a}$, where we use indices $\alpha, \beta, \gamma, \ldots$ for $\mathbf{m}$ space and indices $a, b, c, \ldots$ for $\mathbf{h}$ space, and $t_{\alpha}$ and $t_{a}$ are corresponding generators. We can write the commutation relations as

$$
\left[t_{a}, t_{b}\right]=f_{a b}^{c} t_{c} \quad\left[t_{a}, t_{\beta}\right]=f_{a \beta}^{\alpha} t_{\alpha} \quad\left[t_{\alpha}, t_{\beta}\right]=f_{\alpha \beta}^{c} t_{c}
$$


Maurer-Cartan equation (101) can be decomposed as

$$
\begin{array}{rrr}
d J^{a}+\frac{1}{2} f_{b c}^{a} J^{b} \wedge J^{c}+\frac{1}{2} f_{\alpha \beta}^{a} J^{\alpha} \wedge J^{\beta}=0 & \text { on } \mathbf{h}-\text { space } \\
d J^{\alpha}+f_{\beta a}^{\alpha} J^{\beta} \wedge J^{a}=0 & \text { on } \mathbf{m}-\text { space }
\end{array}
$$

We can also decompose Cartan structural equations. Decomposition of first structural equation gives us

$$
\begin{array}{rr}
d w^{a}+w_{b}^{a} \wedge w^{b}+w_{\alpha}^{a} \wedge w^{\alpha}=0 & \text { on } \mathbf{h}-\text { space } \\
d w^{\alpha}+w_{\beta}^{\alpha} \wedge w^{\beta}+w_{a}^{\alpha} \wedge w^{a}=0 & \text { on } \mathbf{m}-\text { space }
\end{array}
$$

comparison of these equations with the Maurer-Cartan equations (113)-(114) gives us the following connections

$$
\begin{aligned}
& w^{a}=J^{a} \quad w_{c}^{a}=\frac{1}{2} f_{b c}^{a} J^{b} \quad w_{\beta}^{a}=\frac{1}{2} f_{\alpha \beta}^{a} J^{\alpha} \\
& w^{\alpha}=J^{\alpha} \quad w_{\beta}^{\alpha}=\frac{1}{2} f_{a \beta}^{\alpha} J^{a} \quad w_{a}^{\alpha}=\frac{1}{2} f_{\beta a}^{\alpha} J^{\beta}
\end{aligned}
$$

Decomposition of second Cartan structural equation leads to the following equations

$$
\begin{aligned}
d w_{b}^{a}+w_{c}^{a} \wedge w_{b}^{c}+w_{\lambda}^{a} \wedge w_{b}^{\lambda}= & \frac{1}{2} R_{b c d}^{a} w^{c} \wedge w^{d}+\frac{1}{2} R_{b c \lambda}^{a} w^{c} \wedge w^{\lambda} \\
& +\frac{1}{2} R_{b \lambda c}^{a} w^{\lambda} \wedge w^{c}+\frac{1}{2} R_{b \lambda \mu}^{a} w^{\lambda} \wedge w^{\mu} \\
d w_{\alpha}^{a}+w_{c}^{a} \wedge w_{\alpha}^{c}+w_{\lambda}^{a} \wedge w_{\alpha}^{\lambda}= & \frac{1}{2} R_{\alpha b c}^{a} w^{b} \wedge w^{c}+\frac{1}{2} R_{\alpha b \beta}^{a} w^{b} \wedge w^{\beta} \\
& +\frac{1}{2} R_{\alpha \beta b}^{a} w^{\beta} \wedge w^{b}+\frac{1}{2} R_{\alpha \lambda \mu}^{a} w^{\lambda} \wedge w^{\mu} \\
d w_{\beta}^{\alpha}+w_{\gamma}^{\alpha} \wedge w_{\beta}^{\gamma}+w_{a}^{\alpha} \wedge w_{\beta}^{a}= & \frac{1}{2} R_{\beta a b}^{\alpha} w^{a} \wedge w^{b}+\frac{1}{2} R_{\beta a \gamma}^{\alpha} w^{a} \wedge w^{\gamma} \\
& +\frac{1}{2} R_{\beta \gamma a}^{\alpha} w^{\gamma} \wedge w^{a}+\frac{1}{2} R_{\beta \lambda \mu}^{\alpha} w^{\lambda} \wedge w^{\mu} \\
d w_{a}^{\alpha}+w_{\gamma}^{\alpha} \wedge w_{a}^{\gamma}+w_{b}^{\alpha} \wedge w_{a}^{b}= & \frac{1}{2} R_{a b c}^{\alpha} w^{b} \wedge w^{c}+\frac{1}{2} R_{a b \lambda}^{\alpha} w^{b} \wedge w^{\lambda} \\
& +\frac{1}{2} R_{a \lambda b}^{\alpha} w^{\lambda} \wedge w^{b}+\frac{1}{2} R_{a \lambda \mu}^{\alpha} w^{\lambda} \wedge w^{\mu}
\end{aligned}
$$


Inserting (117) and (118) into (119) gives the following curvature components

$$
\begin{aligned}
R_{b d e}^{a} & =\frac{1}{2}\left(f_{d c}^{a} f_{e b}^{c}-f_{c b}^{a} f_{d e}^{c}\right)=\frac{1}{2} f_{c e}^{a} f_{b d}^{c} \\
R_{b \alpha \beta}^{a} & =\frac{1}{2}\left(f_{\alpha \lambda}^{a} f_{\beta b}^{\lambda}-f_{c b}^{a} f_{\alpha \beta}^{c}\right)=\frac{1}{2} f_{\lambda \beta}^{a} f_{b \alpha}^{\lambda} \\
R_{b c \lambda}^{a} & =R_{b \lambda c}^{a}=0
\end{aligned}
$$

where we used the jacobi identity $f_{c[d}^{a} f_{b e]}^{c}=0$ in (123), and $f_{\lambda \alpha}^{a} f_{b \beta}^{\lambda}+f_{c b}^{a} f_{\beta \alpha}^{c}+$ $f_{\lambda \beta}^{a} f_{\alpha b}^{\lambda}$ in (124). Likewise (120) gives the following curvature components

$$
\begin{aligned}
R_{\alpha c \lambda}^{a} & =\frac{1}{2}\left(f_{c d}^{a} f_{\lambda \alpha}^{d}-f_{\beta \alpha}^{a} f_{c \lambda}^{\beta}\right)=\frac{1}{2} f_{\beta \lambda}^{a} f_{\alpha c}^{\beta} \\
R_{\alpha \lambda c}^{a} & =\frac{1}{2}\left(f_{\lambda \beta}^{a} f_{c \alpha}^{\beta}-f_{\beta \alpha}^{a} f_{\lambda c}^{\beta}\right)=\frac{1}{2} f_{b c}^{a} f_{\alpha \lambda}^{b} \\
R_{\alpha b c}^{a} & =R_{\alpha \lambda \mu}^{a}=0
\end{aligned}
$$

where we used the jacobi identity $f_{d c}^{a} f_{\alpha \lambda}^{d}+f_{\beta \alpha}^{a} f_{\lambda c}^{\beta}+f_{\beta \lambda}^{a} f_{c \alpha}^{\beta}=0$ in (126), and $f_{\beta \lambda}^{a} f_{\alpha c}^{\beta}+f_{\beta \alpha}^{a} f_{c \lambda}^{\beta}+f_{b c}^{a} f_{\lambda \alpha}^{b}=0$ in (127). Equation (121) produces the following curvature components

$$
\begin{aligned}
R_{\beta b c}^{\alpha} & =\frac{1}{2}\left(f_{b \gamma}^{\alpha} f_{c \beta}^{\gamma}-f_{a \beta}^{\alpha} f_{b c}^{a}\right)=\frac{1}{2} f_{\gamma c}^{\alpha} f_{\beta b}^{\gamma} \\
R_{\beta \lambda \mu}^{\alpha} & =\frac{1}{2}\left(f_{\lambda a}^{\alpha} f_{\mu \beta}^{a}-f_{a \beta}^{\alpha} f_{\lambda \mu}^{a}\right)=\frac{1}{2} f_{a \mu}^{\alpha} f_{\beta \lambda}^{a} \\
R_{\beta a \gamma}^{\alpha} & =R_{\beta \gamma a}^{\alpha}=0
\end{aligned}
$$

where we used the jacobi identity $f_{\gamma b}^{\alpha} f_{\beta c}^{\gamma}+f_{a \beta}^{\alpha} f_{c b}^{a}+f_{\gamma c}^{\alpha} f_{b \beta}^{\gamma}=0$ in (129), and $f_{a \lambda}^{\alpha} f_{\beta \mu}^{a}+f_{a \beta}^{\alpha} f_{\mu \lambda}^{a}+f_{a \mu}^{\alpha} f_{\lambda \beta}^{a}=0$ in (130). Finally, equation (122) gives the following curvature components

$$
\begin{aligned}
R_{a c \lambda}^{\alpha} & =\frac{1}{2}\left(f_{c \beta}^{\alpha} f_{\lambda a}^{\beta}-f_{\beta a}^{\alpha} f_{c \lambda}^{\beta}\right)=\frac{1}{2} f_{b \lambda}^{\alpha} f_{a c}^{b} \\
R_{a \lambda c}^{\alpha} & =\frac{1}{2}\left(f_{\lambda b}^{\alpha} f_{c a}^{b}-f_{\beta a}^{\alpha} f_{\lambda c}^{\beta}\right)=\frac{1}{2} f_{\beta c}^{\alpha} f_{a \lambda}^{\beta} \\
R_{a b c}^{\alpha} & =R_{a \lambda \mu}^{\alpha}=0
\end{aligned}
$$

where we used the jacobi identity $f_{\beta c}^{\alpha} f_{a \lambda}^{\beta}+f_{\beta a}^{\alpha} f_{\lambda c}^{\beta}+f_{b \lambda}^{\alpha} f_{c a}^{b}=0$ in (132), and $f_{b \lambda}^{\alpha} f_{a c}^{b}+f_{\beta a}^{\alpha} f_{c \lambda}^{\beta}+f_{\beta c}^{\alpha} f_{\lambda a}^{\beta}=0$ in (133). Obviously we can write similar equations with tilde. 
We want to write down curvature relations between symmetric spaces ( $\mathbf{m}$ and $\tilde{\mathbf{m}}$ ) and corresponding closed spaces $(\mathbf{h}$ and $\tilde{\mathbf{h}})$ on $\mathbf{g}$ and $\tilde{\mathbf{g}}$. To realize this objective we will use the bracket relations derived from pseudoduality equations. In case of non-mixing pseudoduality, we will make use of bracket relation (83)-(86). After eliminating $A_{-}$and $k_{-}$terms we obtain the following relations between connection one forms

$$
\begin{array}{rlrl}
\tilde{w}_{a}^{\alpha} & =w_{a}^{\alpha} & \tilde{w}_{\beta}^{\alpha}=w_{\beta}^{\alpha} \\
\tilde{w}_{\beta}^{a}=w_{\beta}^{a} & \tilde{w}_{b}^{a}=w_{b}^{a}
\end{array}
$$

where we used the definitions (117) and (118) for the connection two forms. Taking exterior derivative of these connections we obtain the result

$$
\tilde{R}_{B C D}^{A}=-R_{B C D}^{A}
$$

where $A, B, C$ and $D$ represent indices corresponding to $M$ or $H$-space elements depending on which equation is used. But curvature expressions found above restrict all curvature components to exist. Therefore we will only have curvatures whose all indices belongs to one space ( $\mathbf{m}$ or $\mathbf{h}$ ) or being shared equally, otherwise they do not exist. On the other hand when we consider mixing pseudoduality, we observe that curvature components mix. From the connection two-forms we obtain the relations

$$
\begin{aligned}
\tilde{w}_{\beta}^{\alpha}+\tilde{w}_{a}^{\alpha} T_{\beta}^{a}(0) & =w_{\beta}^{\alpha}+T_{a}^{\alpha}(0) w_{\beta}^{a} \\
\tilde{w}_{\beta}^{\alpha} T_{b}^{\beta}(0)+\tilde{w}_{b}^{\alpha} & =T_{a}^{\alpha}(0) w_{b}^{a}+w_{b}^{\alpha} \\
\tilde{w}_{b}^{a}+\tilde{w}_{\beta}^{a} T_{b}^{\beta}(0) & =w_{b}^{a}+T_{\beta}^{a}(0) w_{b}^{\beta} \\
\tilde{w}_{b}^{a} T_{\beta}^{b}(0)+\tilde{w}_{\beta}^{a} & =T_{\gamma}^{a}(0) w_{\beta}^{\gamma}+w_{\beta}^{a}
\end{aligned}
$$

It is clear that once mixing isometries disappear we have (135) and (136). Therefore curvature relations will be

$$
\begin{aligned}
& \hat{R}_{B \mu \nu}^{A}=-\left(\overline{\tilde{R}}_{B \mu \nu}^{A}+\overline{\tilde{R}}_{B \mu c}^{A} T_{\nu}^{c}(0)+\overline{\tilde{R}}_{B c \nu}^{A} T_{\mu}^{c}(0)+\overline{\tilde{R}}_{B c d}^{A} T_{\mu}^{c}(0) T_{\nu}^{d}(0)\right) \\
& \hat{R}_{B \mu d}^{A}=-\left(\overline{\tilde{R}}_{B \mu d}^{A}+\overline{\tilde{R}}_{B c d}^{A} T_{\mu}^{c}(0)+\overline{\tilde{R}}_{B \mu \nu}^{A} T_{d}^{\nu}(0)+\overline{\tilde{R}}_{B c \nu}^{A} T_{\mu}^{c}(0) T_{d}^{\nu}(0)\right) \\
& \hat{R}_{B c \nu}^{A}=-\left(\overline{\tilde{R}}_{B c \nu}^{A}+\overline{\tilde{R}}_{B c d}^{A} T_{\nu}^{d}(0)+\overline{\tilde{R}}_{B \mu \nu}^{A} T_{c}^{\mu}(0)+\overline{\tilde{R}}_{B \mu d}^{A} T_{c}^{\mu}(0) T_{\nu}^{d}(0)\right) \\
& \hat{R}_{B c d}^{A}=-\left(\overline{\tilde{R}}_{B c d}^{A}+\overline{\tilde{R}}_{B \mu d}^{A} T_{c}^{\mu}(0)+\overline{\tilde{R}}_{B c \mu}^{A} T_{d}^{\mu}(0)+\overline{\tilde{R}}_{B \mu \nu}^{A} T_{c}^{\mu}(0) T_{d}^{\nu}(0)\right)
\end{aligned}
$$

where we defined $\hat{R}_{\lambda \mu \nu}^{\alpha} \equiv R_{\lambda \mu \nu}^{\alpha}+T_{a}^{\alpha}(0) R_{\lambda \mu \nu}^{a}$ and $\overline{\tilde{R}}_{\lambda \mu \nu}^{\alpha} \equiv \tilde{R}_{\lambda \mu \nu}^{\alpha}+\tilde{R}_{b \mu \nu}^{\alpha} T_{\lambda}^{b}(0)$, and $A, B$ represent indices for $\mathbf{m}$ or $\mathbf{h}$-spaces. Obviously if all mixing parts are set to zero we obtain the simplest case (137). 


\section{One Loop Renormalization Group $\beta$-function}

It is noted that renormalization group $\beta$-function to one-loop order [26] is given by

$$
\beta_{m n}=\frac{R_{m n}}{2 \pi}
$$

where $R_{m n}$ is Ricci curvature of connections $w_{j}^{i}$. On $\mathbf{g}$ it is written as

$$
\beta_{i j}=\frac{1}{4 \pi} f_{n j}^{k} f_{i k}^{n}
$$

On decomposed spaces $\mathbf{h}$ and $\mathbf{m}$ one loop $\beta$-functions will be

$$
\begin{aligned}
& \beta_{a b}=\frac{1}{4 \pi}\left(f_{\beta b}^{\alpha} f_{a \alpha}^{\beta}+f_{d b}^{c} f_{a c}^{d}\right) \\
& \beta_{\alpha \gamma}=\frac{1}{4 \pi}\left(f_{\lambda \gamma}^{a} f_{\alpha a}^{\lambda}+f_{\alpha \lambda}^{a} f_{a \gamma}^{\lambda}\right)
\end{aligned}
$$

It is readily observed that $R_{a \alpha}=R_{\alpha a}=0$. On pseudodual spaces one can write the following relations

$$
\beta_{i j}=-\tilde{\beta}_{i j} \quad \beta_{a b}=-\tilde{\beta}_{a b} \quad \beta_{\alpha \gamma}=-\tilde{\beta}_{\alpha \gamma}
$$

if there is a non-mixing pseudoduality. On the other hand if there is a mixing pseudoduality we have

$$
\begin{aligned}
& \beta_{a b}=-\tilde{\beta}_{a b}-\tilde{\beta}_{a \nu} T_{b}^{\nu}(0)-\tilde{\beta}_{\nu b} T_{a}^{v}(0)-\tilde{\beta}_{\mu \nu} T_{a}^{\mu}(0) T_{b}^{\nu}(0) \\
& \beta_{\mu \nu}=-\tilde{\beta}_{\mu \nu}-\tilde{\beta}_{d \nu} T_{\mu}^{d}(0)-\tilde{\beta}_{\mu d} T_{\nu}^{d}(0)-\tilde{\beta}_{a b} T_{\mu}^{a}(0) T_{\nu}^{b}(0)
\end{aligned}
$$

where we defined $\tilde{\beta}_{\nu b} \equiv \frac{1}{2 \pi}\left\{\tilde{R}_{\nu \mu b}^{c} T_{c}^{\mu}(0)+\tilde{R}_{\nu c b}^{\mu} T_{\mu}^{c}(0)\right\}, \tilde{\beta}_{a \nu} \equiv \frac{1}{2 \pi}\left\{\tilde{R}_{a \mu \nu}^{c} T_{c}^{\mu}(0)+\right.$ $\left.\tilde{R}_{a c \nu}^{\mu} T_{\mu}^{c}(0)\right\}, \tilde{\beta}_{d \nu} \equiv \frac{1}{2 \pi}\left\{\tilde{R}_{d \lambda \nu}^{c} T_{c}^{\lambda}(0)+\tilde{R}_{d c \nu}^{\lambda} T_{\lambda}^{c}(0)\right\}$ and $\tilde{\beta}_{\mu d} \equiv \frac{1}{2 \pi}\left\{\tilde{R}_{\mu \lambda d}^{c} T_{c}^{\lambda}(0)+\right.$ $\left.\tilde{R}_{\mu c d}^{\lambda} T_{\lambda}^{c}(0)\right\}$ on the contrary to (146). We notice that if all mixing isometries vanish, then we get (150). We notice that we will also obtain additional mixing components of $\beta$-function, but we avoid to obtain them.

\section{Discussion}

In this section we were able to obtain infinite number of pseudoduality equations by switching from Lie group expressions to Lie algebra ones. We observed that pseudoduality transformation respects the conservation law of 
currents. To understand what these currents imply for let us write pseudoduality equations as

$$
\begin{aligned}
& \tilde{J}_{+}^{(L)}=+T J_{+}^{(L)} \\
& \tilde{J}_{-}^{(L)}=-T J_{-}^{(L)}
\end{aligned}
$$

where $J_{ \pm}^{(L)}=g^{-1} \partial_{ \pm} g$. First equation implies that $T$ is a function of $\sigma^{+}$ as above. Second equation is interesting and gives the information about currents. If we take $\partial_{+}$of second equation we obtain that

$$
\left[\tilde{g}^{-1} \partial_{-} \tilde{g}, \tilde{g}^{-1} \partial_{-} \tilde{g}\right]_{\tilde{G}}=-\left(\partial_{+} T\right)\left(g^{-1} \partial_{-} g\right)-T\left[g^{-1} \partial_{-} g, g^{-1} \partial_{+} g\right]_{G}
$$

We notice that $g^{-1} \partial_{ \pm} g \in \mathbf{g}$, and if we use the definition $\operatorname{ad}_{\mathbf{g}}(X)(Y)=[X, Y]_{G}$ this equation can be written as

$$
\operatorname{ad}_{\tilde{\mathbf{g}}}\left(\tilde{J}_{+}^{(L)}\right)\left(\tilde{J}_{-}^{(L)}\right)=\left(\partial_{+} T\right) J_{-}^{(L)}+\operatorname{Tad}_{\mathbf{g}}\left(J_{+}^{(L)}\right)\left(J_{-}^{(L)}\right)
$$

If the second pseudoduality equation is inserted then one gets

$$
-\operatorname{ad}_{\tilde{\mathbf{g}}}\left(\tilde{J}_{+}^{(L)}\right) T-\operatorname{Tad}_{\mathbf{g}}\left(J_{+}^{(L)}\right)=\left(\partial_{+} T\right)
$$

It is obvious that this is the lie algebra version of the $A d G \times A d \tilde{G}$ action on T. $\operatorname{ad}_{\mathbf{g}}\left(J_{+}^{(L)}\right)$ is the orthogonal flat connection on $g^{*} T G$ as defined in section (4). One may find curvature relations using these connections as above. Thus another interpretation of pseudoduality is that since $J_{+}^{(L)}$ depends only on $\sigma^{+}$, so does $T$. Hence if we define a parallel transport $P(\sigma)$ from $(0,0)$ to $\sigma=\left(\sigma^{+}, \sigma^{-}\right)$, pseudoduality equations may be written as

$$
*_{\Sigma}(\tilde{P}(\sigma))^{-1}\left(\tilde{g}^{-1} d \tilde{g}\right)=T(0)\left(P(\sigma)^{-1} g^{-1} d g\right)
$$

where $T(0)=\tilde{P}(\sigma) T(\sigma) P^{-1}(\sigma)$. This means that we start with $g^{-1} d g$, and parallel transport it to origin, and do the same on the dual model. We finally use the fixed isometry $T(0)$ to equate these two fields at the origins.

\section{Acknowledgments}

I would like to thank O. Alvarez for his comments, helpful discussions, and reading an earlier draft of the manuscript. I would like to thank E. A. Ivanov for bringing his important paper to my attention. 


\section{References}

[1] T. Curtright and C.Zachos, Currents, charges, and canonical structure of pseudochiral models, Phys. Rev. D49 (1994) 5408-5421, hep-th/9401006/.

[2] E. A. Ivanov, Duality in $d=2$ sigma models of chiral field with anomaly, Theor. Math. Phys. 71 (1987) 474-484.

[3] O. Alvarez, Target space pseudoduality between dual symmetric spaces, Nucl. Phys. B582 (2000) 139, hep-th/0004120/.

[4] O. Alvarez, Pseudoduality in Sigma Models, Nucl.Phys. B638 (2002) 328350, hep-th/0204011/.

[5] M.Sarisaman, Pseudoduality and Conserved Currents in Sigma Models, Mod. Phys. Lett. A, Vol. 24, No. 2 (2009) pp. 123-134.

[6] S. Helgason, Differential Geometry, Lie Groups, and Symmetric Spaces, Academic Press, 1978.

[7] B.O’Neill, Semi-Riemannian Geometry, Academic Press, 1983.

[8] J.A. Wolf, Spaces of Constant Curvature, Publish or Perish, Inc., fifth ed., 1984.

[9] A. Arvanitoyeorgos, An Introduction to Lie Groups and the Geometry of Homogeneous Spaces, American Mathematical Society, Student Mathematical Library 22, 2003.

[10] H. Eichenherr, M. Forger, Higher local conservation laws for nonlinear sigma models on symmetric spaces, Commun. Math. Phys. 82 (1981) 227.

[11] H. Eichenherr, M. Forger, On the dual symmetry of the non-linear sigma models, Nucl. Phys. B 155 (1978) 381.

[12] H. Eichenherr, M. Forger, More about non-linear sigma models on symmetric spaces, Nucl. Phys. B 164 (1980) 528.

[13] H. Eichenherr, M. Forger, Nucl. Phys. B 282 (1987) 745, Erratum.

[14] J.M. Evans, A.J. Mountain, Commuting charges and symmetric spaces, Physics Letters B, v. 483, iss. 1-3, p. 290-298. 
[15] P. Fendley, Sigma models as perturbed conformal field theories, Phys. Rev. Lett. 83 (1999) 4468, hep-th/9906036/.

[16] J.H. Schwarz, Classical symmetries of some two-dimensional models, Nucl. Phys. B447 (1995) 137, hep-th/9503078/.

[17] J.H. Schwarz, Classical symmetries of some two-dimensional models coupled to gravity, Nucl. Phys. B454 (1995) 427, hep-th/9506076/.

[18] O.A. Castro Alvaredo, J.L. Miramontes, Massive symmetric space sine-Gordon soliton theories and perturbed conformal field theory, hep-th/0002219/.

[19] C.R. Fernandez-Pousa, M.V. Gallas, T.J. Hollowood and J.L. Miramontes, The symmetric space and homogeneous sine-Gordon theories, Nucl. Phys. B484 (1997) 609, hep-th/9606032/.

[20] I. Bakas, Q-H. Park, H-J. Shin, Lagrangian formulation of symmetric space sine-Gordon models, Phys. Lett. B372 (1996) 45, hep-th/9512030/.

[21] J.M. Evans, D. Kagan and C.A.S. Young, Non-local charges and the quantum integrability of sigma models on the symmetric spaces $S O(2 n) / S O(n) x S O(n)$ and $S p(2 n) / S p(n) x S p(n)$, Physics Letters B 597 (2004) 112.

[22] J.M. Evans, on symmetric spaces, Nuclear Physics B 717 (2005) 327.

[23] J.M. Evans, D. Kagan, N.J. MacKay and C.A.S. Young, Quantum higher-spin local charges in symmetric space sigma models, Journal of High Energy Physics 0501 (2005) 020.

[24] M.Sarisaman, Pseudoduality In Supersymmetric Sigma Models on Symmetric Spaces, hep-th/0904.4671/.

[25] E. Witten, Nonabelian bozonization in two dimensions, Commun. Math. Phys. 92 (1984) 455-472.

[26] S. V. Ketov, Quantum Non-Linear Sigma-Models: From Quantum Field Theory to Supersymmetry, Conformal Field Theory, Black Holes and Strings, Texts and Monographs in Physics, Springer, Berlin, Germany, 2000 . 